\title{
On the problem of the relation between phason elasticity and phason dynamics in quasicrystals
}

\author{
Gerrit Coddens \\ Laboratoire des Solides Irradiés, Ecole Polytechnique, \\ F-91128-Palaiseau CEDEX, France
}

(Dated: today)

\begin{abstract}
It has recently been claimed that the dynamics of long-wavelength phason fluctuations has been observed in $i$-AlPdMn quasicrystals. [1] We will show that the data reported call for a more detailed development of the elasticity theory of Jarić and Nelsson [2] in order to determine the nature of small phonon-like atomic displacements with a symmetry that follows the phason elastic constants. We also show that a simple model with a single diffusing tile is sufficient to produce a signal that (1) is situated at a "satellite position" at a distance $q$ from each Bragg peak, that (2) has an intensity that scales with the intensity of the corresponding Bragg peak, (3) falls off as $1 / q^{2}$ and (4) has a time decay constant that is proportional to $1 / D q^{2}$. It is thus superfluous to call for a picture of "phason waves" in order to explain such data, especially as such "waves" violate many physical principles.
\end{abstract}

\section{INTRODUCTION}

It has recently been claimed that the dynamics of long-wavelength phason fluctuations has been observed in $i$ AlPdMn quasicrystals. 11 These claims were based on the observation of very long relaxation times in the so-called speckle patterns of the diffuse scattering measured with coherent X-rays. We report here the results of a number of investigations and model calculations on coherent scattering signals for phason dynamics, that show that there are problems with these claims. Let us resume some important points of our paper:

(1) There is no proof that the (kinetics of the) diffuse scattering observed in these data is due to tile disorder produced by atomic jumps. Alternative interpretations for the data are possible and have not been ruled out. Chemical disorder and a field of small atomic displacements that are not phason jumps can lead to very similar signals.

(2) Atomic jumps are in principle not correlated over long distances, especially when they participate in atomic self-diffusion. Experimental data never code correlations between atomic jumps. They correspond to correlations between atomic positions. Most importantly, the diffusion of a single tile already shares many features with the signal reported, while it does not involve any correlations whatsoever.

(3) Identifying the diffuse scattering with jumps is in contradiction with quasielastic neutron scattering data which clearly demonstrate that the number of jumps increases with $\mathrm{T}$, while the diffuse scattering shows exactly the opposite behaviour.

(4) Introducing a concept of a phason as a displacive mode of atomic jumps in a QC is problematic. An ondulating cut ("sine wave") does not produce a physical wave in physical space. The displacement field defined by the "sine wave" is non-periodic, and the concept of such a mode introduces notions that are unphysical, such as spooky longdistance correlations between isolated phason jumps. The "sine wave" is in this respect not any better than a rigid translation of the cut.

(5) One has confused coherent scattering signals with evidence for collective effects, Fourier components with physical waves, the Fourier transform of the QC with the Fourier transform of a field of atomic jumps.

(6) One has overinterpreted Lubensky's statement 3 that phasons are diffusive.

(7) The available data are not specific enough to warrant claims that they would present evidence for the random tiling model.

(8) Contrary to statements by several authors, the terminology "phason jumps" is by all standards a correct terminology.

(9) The authors analyze the $Q$-dependence of the fluctuating signal as though it would be a static structure factor, while this can never be correct as the dynamical signal they have measured is governed by an intrinsically different dynamical structure factor. More precisely their photon correlation spectroscopy measurements probe the dynamics in terms of the intermediate scattering function $I(\mathbf{Q}, t)$. That at a given value of $\mathbf{Q}$ this intermediate scattering function is of the form to $I(\mathbf{Q}) e^{-t / \tau_{0}}$, where $I(\mathbf{Q})$ more or less follows the trends of the static structure factor $S(\mathbf{Q})$, does not at all warrant that the interpretation and the calculation of $S(\mathbf{Q})$ could be applied as valid procedures for the calculation and the interpretation of $I(\mathbf{Q})$.

Several of these problems are quite subtle and require a detailed discussion. The task is rendered difficult by interferences between the problems listed above. The paper is organized as follows. In the second Section we prove that the "sine wave" proposed by the authors is not a proper wave, and that it is not periodic. We take the occasion to show that the interpretation of the data is not unique. In the third Section we show why the 
coherence of the data does not imply that there would be correlations between atomic jumps. In the fourth Section we explain why the neutron data rule out the scenario of tile flips proposed by the authors. In Section $\mathrm{V}$ we discuss a number of problems for the understanding of the elasticity theory. With respect to point (9) we show in Section VI what a correct methodology of approach to coherent quasielastic scattering signals from phason dynamics should be. We discuss several models and derive here an extremely important result: A simple model with a single diffusing tile is sufficient to produce a signal that (1) is situated at "satellite positions" at a distance $q$ from each Bragg peak, that (2) has an intensity that scales with the intensity of the corresponding Bragg peak, (3) falls off as $1 / q^{2}$ and (4) has a time decay constant that is proportional to $1 / D q^{2}$. In Section VII we reexamine the evidence presented in favour of the random tiling interpretation. In Section VIII we discuss the validity of the claim that "phason jumps" would not be a correct terminology. And in Section IX we conclude.

\section{PERIODIC DELUSIONS}

\section{A. The function $u_{\perp} \sin \left(q_{\|} x_{\|}\right)$is not a wave}

Various authors [1, [4] have proposed "phason waves" of the type $u_{\perp} \sin \left(q_{\|} x_{\|}\right)$, with wavelength $\lambda=2 \pi / q_{\|}$along parallel space $E_{\|}$, and polarisation with an amplitude $u_{\perp}$ along perpendicular space $E_{\perp}$. But it is an obvious mathematical fact that this does not define a periodic displacement wave in parallel space $E_{\|}$. To see and convince oneself of this, it suffices to inspect the displacement pattern the "sine wave" brings about on the drawing that defines the cut-and-projection algorithm in $E=E_{\|} \oplus E_{\perp}=\mathbb{R}^{2}$ that generates the Fibonacci sequence. The very misleading notation as a perfect sine wave $u_{\perp} \sin \left(q_{\|} x_{\|}\right)$creates the illusion that there is a periodicity by definition. Certainly, the values this function takes in $E_{\perp}$ are periodic. But this is not the end of the story, as we have to inspect how this is translated by the algorithm into a displacement field in $E_{\|}$. With respect to this issue, the value the sine function takes in perpendicular space is not the only variable that comes into play. What also comes into play at a given value of $x_{\|} \in E_{\|}$is the position of the atomic surface $\mathcal{W}$ at $\left(x_{\|}, x_{\perp}\right)$ with respect to the cut. This position will define the minimum value $t\left(x_{\|}\right)$of perpendicular space ondulation it takes to induce a jump. Let us call the end points of $\mathcal{W}$, $A$ and $B$. We take $A$ to be the point with the higher $x_{\perp}$-value. The minimum value $t\left(x_{\|}\right)$is the distance between the point where parallel space cuts the atomic surface and one of the endpoints $A$ or $B$ of that atomic surface. It is the comparison between $t\left(x_{\|}\right)$and $u_{\perp} \sin \left(q_{\|} x_{\|}\right)$, rather than the value $u_{\perp} \sin \left(q_{\|} x_{\|}\right)$alone that will determine if there is a jump or otherwise. The function $t\left(x_{\|}\right)$is quasiperiodic. Let us note the disordered QC generated by the sine wave $u_{\perp} \sin \left(q_{\|} x_{\|}\right)$as $\mathbb{Q} \mathbb{C}^{*}$, and the perfect $\mathrm{QC}$ as $\mathbb{Q} \mathbb{C}$. To give $\mathbb{Q C}^{*}$ as a whole a periodic description through a superspace embedding one needs to lift it to dimension 3. This is obvious from the fact that the Fourier spectrum of $\mathbb{Q} \mathbb{C}^{*}$ is spanned by three reciprocal lattice vectors, viz. the two reciprocal lattice vectors that span the Fourier spectrum of the Fibonacci lattice $\mathbb{Q C}$ and the vector $q_{\|}$. Note that when we calculate the Fourier spectrum of $\mathbb{Q} \mathbb{C}^{*}$ to better approximations, by taking in higher order terms of the Taylor expansion that is used in the calculation (see below), the harmonics with wave vectors $2 q_{\|}, 3 q_{\|}$, etc... will enter, such that the statement that $q_{\|}$is a third generator of the Fourier spectrum is perfectly accurate (see below).

The Fourier spectrum we are talking about here, is the one of the modified QC, i.e. $\mathbb{Q} \mathbb{C}^{*}$, which is of course different from the Fourier spectrum of the displacement field $\mathbb{D}$ of phason jumps. The Fourier spectrum of the jump field $\mathbb{D}$ itself is not an experimentally measured quantity. The field $\mathbb{D}$ is not at all periodic, as we show now on the example of the Fibonacci chain.

The cut in the superspace description of the Fibonacci chain can pass either through zero or through two endpoints of atomic surfaces. In fact, if there is at least one, it means that the cut is exactly at the position of inducing a jump. The other end point of that jump will then also be an endpoint of an atomic surface, viz. of that atomic surface one is swapping to. When the endpoint of the first atomic surface is of the type $\mathrm{A}$, then the endpoint on the second atomic surface will be of the type B, because all this together defines a single jump. There will be no endpoint of any other atomic surface on the cut, because else the slope of the cut would be rational. This shows that the number of atomic surface endpoints can only be 0 or 2 , and that when there are two of them, they are separated by a jump distance, because they define a single jump and there can be only one jump at a time in the cut. It is therefore possible to take a point $O$ on the cut, such that, to the right of it, the cut does not contain a single endpoint of an atomic surface. Let us take $O$ as the origin of the $x_{\|}$coordinates.

Choose an arbitrary large positive integer number $K \in \mathbb{N}$. Consider now the interval $[0, K \lambda]$ on parallel space. Consider the atomic positions $x_{j}$ in this interval. In each point $x_{j}$, it will take a minimal non-zero translation $t_{j} \neq 0$ of the cut along $E_{\perp}$ to induce an atomic jump, because, by our choice of $[0, K \lambda]$, we know that in $x_{j}$ the cut does not pass through the end point of an atomic surface. For all the atomic positions $x_{j}$ within $[0, K \lambda]$ we can define such a minimal non-zero translation $t_{j}$. The finite set of strictly positive numbers $\left|t_{j}\right|, j \geq 0$ does not contain 0 , such 
that it has a strictly positive minimum value. Call $a$ this minimum, and take $0<\left|u_{\perp}\right|<a$. Then $u_{\perp} \sin \left(q_{\|} x_{\|}\right)$will not induce a single atomic jump over the whole interval $[0, K \lambda]$. By taking increasing values of $K$ we can make this situation arbitrarily bad. In fact, $a$ functions as a density parameter for the jump field. Nevertheless, the "sine wave" does define a non-zero displacement field, because the Fourier spectrum of $\mathbb{Q} \mathbb{C}^{*}$ is different from the Fourier spectrum of $\mathbb{Q C}$.

This argument clearly indicates where the logical error lies in the reasoning of people who think that $u_{\perp} \sin \left(q_{\|} x_{\|}\right)$ would define a periodic displacement field. They have focused on the blind spot of the formal appearance of the "sine wave", without thinking about the way it cuts through the quasiperiodic pattern of the atomic surfaces.

It is obvious from the previous that $\lambda$ has not the meaning of a wavelength for the displacement field $\mathbb{D}$ (see also below). The value $a$ is a measure for the density of jumps on parallel space. Of course, the density is inversely proportional to the average distance between two next neighbour jumps. As the jumps all involve the same jump distance $(\tau-1) / \sqrt{2+\tau}$, this value defines the maxima of the displacement field defined by the sine wave. The value of this maximum amplitude $(\tau-1) / \sqrt{2+\tau}$ cannot be changed by varying $u_{\perp}$. Hence we see that varying the amplitude of the "sine wave" changes the average distance between the maxima of the displacement field, rather than its amplitude. That is certainly not a wave: When we change the amplitude of a true wave, it is exactly the opposite that happens, viz. the distance between its maxima remains the same, while the amplitude of its maxima varies! In fact, $u_{\perp}$ does not act as an amplitude of the displacement field. It rather defines its density (or the average distance between displacements). The fact that the maximum amplitude of the physical displacement field is forcedly $(\tau-1) / \sqrt{2+\tau}$ is already sufficient to provide us with a handwaving argument for the fact that $u_{\perp}$ acts as a density parameter. It is obvious that when $u_{\perp}$ increases, the effects must become stronger. As the only two possible local values for the displacement field are 0 and $(\tau-1) / \sqrt{2+\tau}, u_{\perp}$ only can act on the number of jumps, not on the maximum amplitude of the field.

Our construction shows that $\lambda$ is not a period of the displacement field. In fact, if $\mathrm{X}$ is the first point to the right of $\mathrm{O}$ where we have a jump, then at position $Y:|Y X|=\lambda$ to the left of $\mathrm{X}$, there is no jump, as it should be if $\lambda$ were a period. In fact, in general there will even be no atomic position at $Y$, because in general $\lambda$ will not correspond to a distance between two points of the Fibonacci sequence. The displacement field has no periodicity at all. In fact, restricting the domain of a periodic function of $\mathbb{R}$ to the aperiodic dicrete set of QC lattice points $\mathbb{Q} \mathbb{C}$ already destroys the periodicity stricto sensu, but the situation is rendered worse by restricting also its range in $\mathbb{R}$ to a set that only contains two values 0 and $(\tau-1) / \sqrt{2+\tau}$. The function is even no longer quasiperiodic with a two-dimensional $\mathbb{Z}$-module because its periodic embedding requires a superspace dimension of 3 rather than 2 . It can thus a priori not be claimed on the basis of an argument of (wrongly) assumed periodicity that its Fourier transform would have non-zero components at $Q_{B}+q_{\|}$, where $Q_{B}$ defines a Bragg peak of the QC, and $q_{\|}$is defined by the relation $2 \pi / \lambda=q_{\|}$. But we shall see below that this is nevertheless true. It is also a priori not obvious that the Fourier decomposition of the "sine wave" would yield $Q_{B}+q_{\|}$-values that all have the same value of $q_{\|}$. But again, this is true.

To save the periodicity, one might argue that one made indeed an error but that this just consisted in expressing one's ideas through the wrong formula. One might argue that a displacement wave of jumps of the type $u_{\|} \sin \left(q_{\|} x_{\|}\right)$ surely can exist. But this is also badly wrong: It is impossible to define any sine wave based on a unique jump distance on the Fibonacci chain, because it would just contradict quasiperiodicity (see our argument about restricting the domains of periodic functions). As we already stated, $\lambda$ will only in very exceptional cases correspond to a distance between two atomic positions of the Fibonacci sequence. Hence the positions $P$ were the atomic jumps $P Q$ would have to take origin will in general fall somewhere in between the atomic positions of the quasicrystal. Moreover, the intensity of the diffuse pattern will not scale with $Q_{\perp, B}^{2}$, when the polarization is along $u_{\|}$.

Finally, we must make a remark about the fact that our approach has been one-dimensional. We can see that our objection can be avoided by taking the direction of the wave vector along the periodic direction of a "two-dimensional" quasicrystal (like a decagonal phase). In that case the "sine wave" does not run into the same conceptual difficulties. We may note that this reminds us of the electron microscopy observations of a "large phason jump" by Edagawa et al. [5], but these authors remain cautious about the interpretation of their data in terms of a "sine wave" [6]. Secondly, domain walls have been observed in icosahedral phases by electron microscopy, with a high density of tile flips. Only a few orientations of such domain walls will be energetically allowed, and it would also require a lot of energy to move them as a whole. The Fourier transform of such a plane is a line. This represents then a correlation in the atomic positions where the flips occur, in the sense that they are all located in the same plane. But the $q_{\|}$vectors that one could associate with the lines mentioned would in the "sine wave" picture correspond to a correlation in the direction normal to the plane, not within it. The domain wall can also not be of the "sine wave" form proposed by the authors, as the displacement field defined by a "sine wave" is not two-dimensional. And within the plane, relaxing phason jumps will not be any more correlated than along a Fibonacci chain (see also below).

In conclusion: There does not exist any true, periodic sine wave of atomic jumps in physical space on a QC. 


\section{B. Calculation of the diffraction pattern of the disordered QC}

We must criticize the way a single Fourier component in the diffuse scattering pattern is given physical meaning in reference [1]. Let us first point out that a Fourier componenent of the diffuse scattering corresponds to a density wave. In the theory of Jaric' and Nelsson, 2 the basic formalism is indeed based on density waves. These density waves are not restricted to the discrete set of atomic positions of the QC as the authors of reference [1] propose in their interpretation, but they are defined on the whole continuum of parallel space, where they are allowed to cancel mutually, etc... In fact this mutual canceling is what happens most of the time because the QC is a discrete density. The diffraction diagram as a whole, with the Bragg peaks and the diffuse scattering, describes thus a priori a field of atomic positions, not a field of atomic jumps.

Let us calculate the diffraction pattern of a Fibonacci chain, whose corresponding two-dimensional "supercrystal" has been modulated (in superspace) by the "sine wave" $u_{\perp} \sin \left(q_{\|} x_{\|}\right)$. After applying the "sine wave" the (nondecorated) two-dimensional lattice will be a truly modulated crystal, whose two-dimensional diffraction pattern will be given by Bragg peaks $\delta\left(\mathbf{Q}-\mathbf{Q}_{B}\right)$ and satellites $\delta\left(\mathbf{Q}-\left(\mathbf{Q}_{B} \pm \mathbf{q}_{\|}\right)\right)$. Let us note the window function of the strip method as $\mathcal{W}$. The points of this modulated lattice that fall into the strip $\mathcal{W} \times E_{\|}$project to the atomic postions of the disordered QC. Alternatively, we can decorate the atomic positions of the modulated "supercrystral" with atomic surfaces $\mathcal{W}$ and cut with $E_{\|}$. What we have to calculate is:

$$
\int e^{\imath \mathbf{Q} \cdot \mathbf{r}} \sum_{(M, N) \in \mathbb{Z}^{2}} \delta\left(\mathbf{r}-\mathbf{r}_{M, N}-\mathbf{u}_{M, N}\right) * \mathcal{W}(\mathbf{r}) d \mathbf{r}=\left(\int e^{\imath \mathbf{Q} \cdot \mathbf{r}} \mathcal{W}(\mathbf{r}) d \mathbf{r}\right) \times\left(\sum_{(M, N) \in \mathbb{Z}^{2}} e^{\imath \mathbf{Q} \cdot\left(\mathbf{r}_{M, N}+\mathbf{u}_{M, N}\right)}\right)
$$

where $\mathbf{u}_{M, N}=\mathbf{u}_{\perp} \sin \left(\mathbf{q}_{\|} \cdot \mathbf{r}_{M, N}\right)$, and where we have indexed the nodes of the "supercrystal" by $(M, N)$. When $u_{\perp}$ is

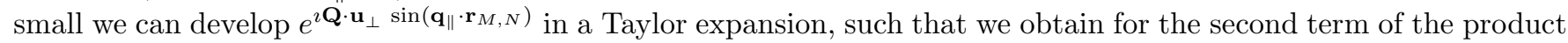
in the righthand side of Eq. (1):

$$
\sum_{(M, N) \in \mathbb{Z}^{2}}\left(e^{\imath \mathbf{Q} \cdot \mathbf{r}_{M, N}}+\left(\frac{\mathbf{Q} \cdot \mathbf{u}_{\perp}}{2}\right)\left(e^{\imath\left(\mathbf{Q}+\mathbf{q}_{\|}\right) \cdot \mathbf{r}_{M, N}}-e^{\imath\left(\mathbf{Q}-\mathbf{q}_{\|}\right) \cdot \mathbf{r}_{M, N}}\right)\right) .
$$

The first exponential will lead to the set of Bragg peaks, the second and third exponentials will lead to Bragg peaks shifted by $\pm \mathbf{q}_{\|}$and further weigthed with $\frac{1}{4}\left(Q_{\perp, B} u_{\perp}\right)^{2}$. We see thus that there will be satellites.

The situation is completely analogous to what we observe with a phonon modulation on a periodic crystal. E.g. a longitudinal phonon displacement field $u \sin (q x)$ on a periodic one-dimensional crystal will introduce satellites at $\pm q$ with respect to each Bragg peak $Q_{B}$. These satellites correspond to density waves with wavelength $2 \pi /\left(q+Q_{B}\right)$. The function $u \sin (q x)$ is defined over the whole of $\mathbb{R}$. By using it to define a displacement wave, we restrict it to the discrete set of the lattice nodes of the crystal. As a corollary its Fourier transform changes from $\delta(Q \pm q)$ for the function whose domain is $\mathbb{R}$, to the infinite set of Dirac measures at $Q_{B} \pm q$. In other words, the price to pay for restricting a continuous function to a lattice, is that one must convolute its Fourier transform with the reciprocal lattice. We see that $2 \pi /\left(Q_{B}+q\right)$ corresponds to a density wave, not to a period, and that we need a whole set of them in order to render the intensity in between the atomic positions of the phonon-modulated crystal zero. The result for the QC follows the same philosphy, with a more complicated reciprocal lattice.

It may look surprizing and counterintuitive that we find that the position of these satellites for the QC is independent from the amplitude $u_{\perp}$, while we have shown that for small enough amplitudes there will be much longer distances between the jumps than given by $2 \pi / q_{\|}$. But this is nevertheless correct (see below). This formalism corresponds to the paradigm used in reference [1]. It corresponds to the idea of Equations [42] and [43] in the paper by Janssen et al.

\section{Criticism of the wave approach}

There are very serious problems with the approach outlined above:

\section{Topological problems}

A problem with the application of the elasticity theory to phason dynamics is that Lubensky's theory is first derived for the example of a structure obtained as a union of two incommensurately modulated sublattices (see his Figure 3.1). As such it creates the impression that he would have avoided the major pitfall of obtaining the excitations from a cut through higher-dimensional dynamics, and the reader thankfully relaxes vigilance. But later on Lubensky lifts the description of the QC to higher-dimensional space. The danger of the idea of a cut through higher-dimensional 
dynamics thus surfaces again, in less alarming appearances by presenting it the other way around. Eventually we find people talking about notions unifying phasons and phonons as being waves that only differ by having different polarization vectors. Between Lubensky's Figure 3.1 and this final formulation there is a big leap with many gaps in the argument.

The phonon dynamics of the Fibonacci chain can in general not be obtained by just making a cut through the phonon dynamics of a two-dimensional lattice, as is suggested by the Equation [42] in the paper of Janssen et al. The phonon problem of the Fibonacci is an unsolved problem of an horrendous difficulty. This has several origins:

(a) The topology of the problem is different from that of the phonon problem on an unbounded lattice. Neighbour interactions with atomic surfaces that are out of the acceptance window, i.e. out of the superspace strip, are just cut away. This gives rise to boundary conditions. This is the discrete analogon of a wave equation on a domain $D$. In the example of the Fibonacci chain with only first neighbour interactions, the boundary problem is quite extreme, as every atomic position is on the boundary $\partial D$ of the domain $D$ of the wave equation, such that $D$ has an empty interior. In fact in $\mathbb{Z}^{2}$ each lattice node has 4 first neighbours, while on the Fibonacci chain each point only has 2 neighbours. This can be formulated as a condition that is the discrete analogon of a boundary condition of the type $\mathbf{e}_{n} \cdot \nabla \psi=0$, on $\partial D$ for the wave equation $\Delta \psi=1 / c^{2} \partial^{2} \psi / \partial t^{2}$; here $\mathbf{e}_{n}$ is the normal to the boundary. But in the discrete problem $\mathbf{e}_{n}$ does not vary smoothly. It takes different orientations, depending on the question if its first neigbour environment corresponds to $L \cdot L, L \cdot S$ or $S \cdot L$ (where $L$ and $S$ are the interatomic distances).

(b) Using another approach, the two-dimensional lattice must be considered to be decorated with atomic surfaces, which implies that there is an infinite number of "atoms" $s$ in the unit cell $(M, N)$. The dynamical matrix that defines all the interactions between pairs of atoms in first-neighbour cells $\left(M, N, s_{1}\right),\left(M+1, N, s_{2}\right)$, etc.., is infinite.

Postulating wavelike solutions as is done e.g. in Eq. [43] of the paper of Janssen et al. is, at least in general, very wrong. There is not the slightest proof that this is reasonable in general. Rigorous studies of the phonon problem on the Fibonacci chain through the transfer matrix method seem to indicate that such an Ansatz is just wrong. This in turn raises the question if there are phason "modes" (in the usual sense of a wave) all together.

A QC is not a continuum but a discrete lattice. The discrete approach prevails thus over the continuum approach. The continuum approach is obtained by taking the long-wavelength limit of the discrete problem. When we propose thus a continuous phonon mode, it must be validated by checking if it makes sense by taking the limit of the discrete solutions. For continuous phonon modes this makes sense. For phasons the situation is all together different:

(a) There are a number of famous papers by Levitov and others [7] that show that in general phasons in QCs crystals are not continuous.

(b) In contrast with what happens with the phonon modes, the displacement field of the postulated phason wave is not at all sinusoidal in parallel space. When we make the discrete graph $u\left(x_{\|}\right)$vs. $x_{\|}$of a long-wavelength phononlike sine wave diplacement field on the Fibonacci chain, and we look at this graph from very far, such that the atomic positions seem to fill a continuum, the picture of the sine wave will become clearly visible. It is this that gives sense to the continuum limit for phonons. When we do the same for the displacement field of the phasonlike "sine wave", we will not be able to make sense of it: In certain regions the graph will appear to consist of two horizontal lines $u\left(x_{\|}\right)=(\tau-1) / \sqrt{2+\tau}$, and $u\left(x_{\|}\right)=0$, as though it would correspond to a two-valued "function". The two lines will have grey shading, rather than being just black. In other regions, it will appear to be just $u\left(x_{\|}\right)=0$ alone. It is thus no longer possible to keep telling accurately what is going on in the QC when we move out to inspect it from further away with a coarsened resolution. Taking the "long-wavelength limit" ceases to be a self-evident concept or method, because (1) one cannot define limits for discontinuous functions, and (2) the displacement field cannot be described or regularized as the restriction of a continuous wave to the $\mathrm{QC}$.

(c) The postulated phason modes lead to notions of long distance coherence between atomic jumps that are completely unphysical. This will be developed below: The closer the jumps are in time, the farther away they must be in space! Also, in all experimental studies of atomic self-diffusion, the paradigm and the evidence is that atomic jumps are not correlated over long distances.

(d) We may add to this that the infinite lattice allows also for modes with an exponential decay in space. These are excluded in a real crystal because these exponential waves diverge, in one direction or another, but in the QC, divergence along the $x_{\perp}$ direction is harmless, due to the restriction to the strip. Hence, if the wavelike solutions were correct, they would lack generality.

\section{Incompleteness of the data}

While in a crystal the spectral reponse for a phonon modulation is rigorously the same at every Bragg peak, in a QC the intensity is different at every Bragg peak. It is thus no longer representative to study a single Fourier component at $Q_{B} \pm q_{\|}$for a given $q_{\|}$-value at a choosen Bragg peak defined by $Q_{B}$. One has a whole set of satellites, with their respective intensities. It would be normal to check experimentally that these relative intensities at $Q_{B} \pm q_{\|}$ for various Bragg peaks are compatible with the calculation of the picture of a superspace phonon. It would also be 
normal to check experimentally if the same $q_{\|}$-value at different Bragg peaks $Q_{B}$ yields identical relaxation times.

\title{
D. Solution of a paradox
}

We must now treat the apparent contradiction that $q_{\|}$does not correspond to a period of the displacement field $\mathbb{D}$, while it appears in the diffraction spectrum.

\author{
1. An important distinction
}

Let us first note that we must make a difference between $\mathbb{Q} \mathbb{C}^{*}$, which is the whole disordered quasicrystal, i.e. a set of atomic positions, and the displacement field $\mathbb{D}$, which is a set of atomic jumps located at certain atomic positions, and which can have a very large average distance between the points where it is non-zero.

\section{The disordered quasicrystal}

It may look paradoxycal that the distance between successive jumps in the displacement field is much larger than the wavelength of the sine wave, while the wave vector $q_{\|}$shows up in the Fourier spectrum of the disordered QC. It is nevertheless true. This is actually no more paradoxical than that the reciprocal lattice vectors of the the $\mathbb{Z}$-module of the superspace embedding (like $2 \pi / L$ and $2 \pi / S$ in the Fibonacci chain) appear (as generators) in the diffraction pattern of the QC, while they are not periods. In fact, we may consider the superspace lattice, decorated with its atomic surfaces as modulated by the sine wave $u_{\perp} \sin \left(q_{\|} x_{\|}\right)$. Following the method of Dewolff, Janssen and Janner, [9] we can approach such a modulation by lifting the reciprocal lattice to a three dimensional periodic lattice. This allows us to see that the Fourier module will now have the additional generator $q_{\|}$. And this does not depend on the amplitude $u_{\perp}$. We can also see that the whole spectrum of harmonics of $q_{\|}$can in principle occur. It is just that they have too weak intensities when they are not observed. E.g. when $u_{\perp}$ becomes larger or if the modulation is not sinusoidal, they may show up. (E.g. if in our derivation of Eq. (2) one develops the exponential to second order, the harmonics $2 q_{\|}$come into play). This can perhaps even be used to find out how we can describe the system without violating the conservation of atoms when the modulation is strong. We can see from this very clearly that $\lambda$ is not a period, just like the interatomic distances $L$ and $S$ in the Fibonacci chain are not periods.

\section{The displacement field}

Let us now check how it is with the paradox for the proper displacement field $\mathbb{D}$. The Fourier spectrum of the displacement field is not the difference of the Fourier spectra of $\mathbb{Q} \mathbb{C}^{*}$ and $\mathbb{Q} \mathbb{C}$; for a displacement from $x_{1}$ to $x_{2}$, $e^{\imath Q\left(x_{2}-x_{1}\right)} \neq e^{\imath Q x_{2}}-e^{\imath Q x_{1}}$, also not in first order. But let us inspect what we obtain when we subtract the Fourier transforms of $\mathbb{Q C}^{*}$ and $\mathbb{Q} \mathbb{C}$. It would yield the Fourier transform of a set of dipoles: There is a negative Dirac measure at each starting point $x_{1}$ of a jump, and a positive Dirac measure at each end point $x_{2}$ of a jump. Hence we have $\mathcal{F}\left[\sum\left(\delta\left(x-x_{2}\right)-\delta\left(x-x_{1}\right)\right)\right]$. What we would need to calculate is $\mathcal{F}\left[\sum(\tau-1) \mathbf{e}_{\|} \delta\left(x-x_{1}\right) / \sqrt{2+\tau}\right]$. In the superspace description the difference spectrum gives rise to just a subtraction of the densities of the atomic surfaces. At one end of an atomic surface the subtraction yields a positive density, in the region where the atomic surfaces do not overlap, at the other end a negative density. When the jump occurs to the right it is the upper end of a subtracted atomic surface where the positive density occurs, and the lower end of the neighbouring subtracted atomic surface where the negative density occurs. We certainly need these pieces of density to make a calculation of the Fourier transform of the displacement field, but we need a method to select only the negative pieces, and weighting it with $\pm(\tau-1) \mathbf{e}_{\|} / \sqrt{2+\tau}$ where the sign depends on the question if the jump occurs to the left or to the right.

To simpfly the problem it is better to consider the two displacement fields $\mathbb{D}_{1}$, defined by $u_{\perp}\left(1+\sin \left(q_{\|} x_{\|}\right)\right)$, and $\mathbb{D}_{2}$ defined by $-u_{\perp}$. The first one will only yield jumps to the right. The second one only jumps to the left. Subtracting the Fourier transform of the perfect QC from the Fourier transform of the QC modulated by $\mathbb{D}_{1}$, will correspond to the Fourier transform $\mathcal{F}_{1}=\mathcal{F}\left(S_{1}\right)$ of a set of dipoles $S_{1}$, that are all oriented to the right. Call the set of origins of the jump vectors of $\mathbb{D}_{1}, B_{1}$, and a dipole oriented to the right $d$. Then $\mathcal{F}_{1}=\mathcal{F}\left(B_{1} * d\right)=\mathcal{F}\left(B_{1}\right) \mathcal{F}(d)$, from which $\mathcal{F}\left(B_{1}\right)$ can be calculated. (There is a problem with this, in that $\mathcal{F}(d)=e^{\imath Q(\tau-1) / \sqrt{2+\tau}}-1$ contains zeros, but these do not occur on the satellites). Similarly we can calculate the Fourier transform of the set $\mathcal{F}\left(B_{2}\right)$ of origins of jump vectors defined by $\mathbb{D}_{2}$. If we take $\mathcal{F}(d)$ to deconvolute, the set $B_{2}$ will be negatively weighted. With this weighting $(\tau-1)\left(\mathcal{F}\left(B_{1}\right)+\mathcal{F}\left(B_{2}\right)\right) / \sqrt{2+\tau}$, will then be the Fourier transform of the displacement field $\mathbb{D}$. When in a given point both a jump to the right occurs within in $\mathbb{D}_{1}$ and a jump to the left within $\mathbb{D}_{2}$, then the two jumps will conveniently add up to zero. In all other points, the jumps will occur with the proper weighting. This point hinges of course on the fact that the amplitude of $u_{\perp}$ must be small enough. If the amplitude of the sine wave is too large, there will be successive jumps of a same atom, and the jump distances will not all be $(\tau-1) / \sqrt{2+\tau}$. In conclusion, 
also for $\mathbb{D}$ the solution of the paradox is that $q_{\|}$does indeed occur in the Fourier spectrum.

\section{Conclusion}

We see that doubling the amplitude $u_{\perp}$ will in first order double the intensity of the satellites both in the Fourier spectrum of $\mathbb{Q} \mathbb{C}^{*}$, and in the Fourier spectrum of the displacement field $\mathbb{D}$. But of course, this does not imply that $\mathbb{Q} \mathbb{C}^{*}$ or $\mathbb{D}$ would be periodic, or that $\mathbb{D}$ would be physically meaninful (see below).

\section{E. Clearly distinguishing the various fields}

Let us resume the situation. A single Fourier component at some value $Q_{B}+q_{\|}$in the diffuse scattering is a continuous density wave and as such cannot be the Fourier transform of a field of phason jumps. Even a set of Fourier components $Q_{B}+q_{\|}$with the same $q_{\|}$-values does not correspond to a periodic wave of jumps:

(a) (If we include also the Bragg peaks,) it just corresponds to a disordered quasicrystal $\mathbb{Q} \mathbb{C}^{*}$, not to the field of jumps that would permit to go from the perfect quasicrystal $\mathbb{Q} \mathbb{C}$ to $\mathbb{Q} \mathbb{C}^{*}$. (Of course the "difference" between the pristine $\mathbb{Q} \mathbb{C}$ and the disordered $\mathbb{Q} \mathbb{C}^{*}$ corresponds to a field of jumps).

(b) We have proved that when a "sine wave" transforms the perfect $\mathbb{Q C}$ into the disordered $\mathbb{Q} \mathbb{C}^{*}$ with such a $q_{\|}$-based set of Fourier components with wave vectors $Q_{B}+q_{\|}$, then this does not imply at all that the transformation from the perfect $\mathbb{Q} \mathbb{C}$ to the disordered $\mathbb{Q} \mathbb{C}^{*}$ would be be based on a periodic wave of atomic jumps at positions of $\mathbb{Q} \mathbb{C}$.

Thus in a diffuse scattering pattern that uniquely corresponds to the disorder generated by tile flips (e.g. in the Monte Carlo simulations of Tang et al. 10, 11]), the intensity at $Q_{B}+q_{\|}$can never have physical meaning, as it corresponds to a continuous sine wave by definition. The diffuse scattering intensity $Q_{B}+q_{\|}$in the disordered-tiling model is only a Fourier component, without any real physical meaning. It corresponds to a density wave for the disordered quasicrystal $\mathbb{Q} \mathbb{C}^{*}$. To obtain a physical meaning we must combine all Fourier components $Q_{B}+q_{\|}$at all Bragg peaks. And in a given point of space, these Fourier components will most of the time just cancel mutually to yield zero density, as the atomic positions are a discrete set. By combining all contributions with wave vectors $Q_{B} \pm q_{\|}$, corresponding to a single $q_{\|}$linearly with their appropriate intensities, we may reconstruct the whole disordered tiling $\mathbb{Q} \mathbb{C}^{*}$, produced by the "sine wave" with wavelength $2 \pi / q_{\|}$from the perfect quasicrystal $\mathbb{Q} \mathbb{C}$.

We see thus that we have the following scheme:

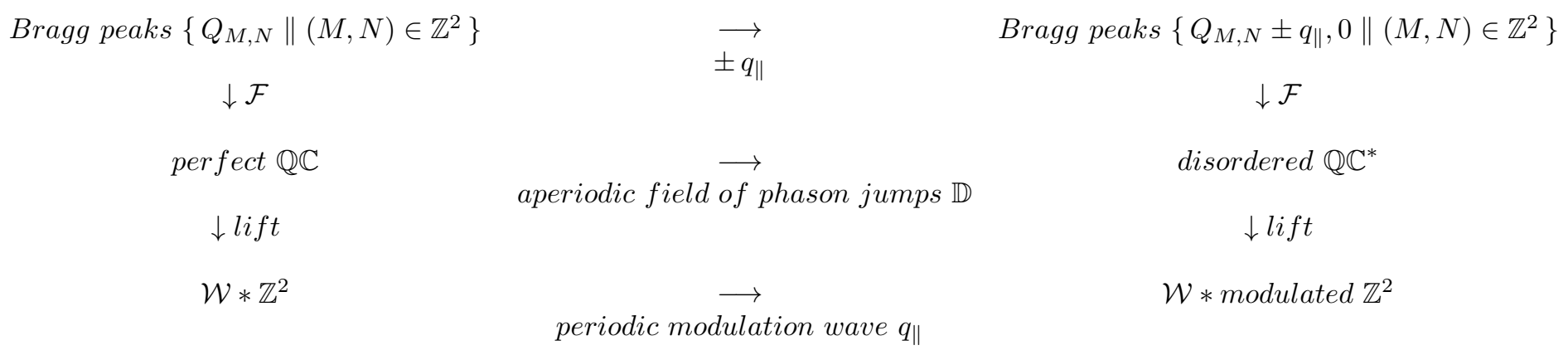

Here only the last line in the diagram corresponds to superspace quantities. The two other lines refer to parallel spaces: The first line to reciprocal space, the second line to direct space.

It is important to realize that the periodic modulation wave is only the Fourier transform of $q_{\|}$in the superspace sense, and that the Fourier transform of $q_{\|}$in $E_{\|}$has no physical meaning, e.g. it does not correspond to the aperiodic field of phason jumps $\mathbb{D}$. The periodicity of the modulation in superspace does not imply that the field of phason jumps $\mathbb{D}$ would be periodic. This field is definitely aperiodic. The field of the phason jumps is not directly measured, and its Fourier transform is not a single value $\delta\left(Q-\left(Q_{B} \pm q_{\|}\right)\right)$. The only quantities that can be directly measured are the ones on the first line of the diagram.

\section{F. Further criticism of the "sine wave" interpretation}

The way the authors analyse the isolated diffuse intensities at $Q_{B}+q_{\|}$by drawing the relaxation time as a function of $q_{\|}$to prove that it would correspond to a diffusive mode of jumps is approximate and incomplete. As we have 
seen it corresponds to a physically meaningless density wave. To obtain meaningful jumps in the case of a sine wave modulation one would have to study a whole set of $Q_{B}+q_{\|}$-values simultaneously, as only a whole set will yield the disordered QC, created by a field of jumps. There is an experimental problem that we do not know exactly how the corresponding amplitudes are to be combined. This is the very same problem as for reconstructing the perfect QC from the intensities of its Bragg peaks. Even if we leave this practical problem aside, there remain conceptual problems.

\section{The postulated diplacement field is unphysical}

We have seen that when $u_{\perp}$ is sufficiently small, $q_{\|}$can correspond to a field that induces very few atomic jumps that are very far away from each other, with no jumps at all in between. If we imagine $u_{\perp}$ as built up from infinitesimal contributions $\delta u_{\perp}$, we can have the sine wave continuously growing from 0 amplitude to the small amplitude $u_{\perp}$. In a given large patch of the QC, we will see then the tile flips occuring consecutively, one by one, and the consective atomic jumps will be separated by very large distances. The closer the phason jumps are in time, the further away they will be in space! That does not evoke an image of a coherent process with correlated jumps! For correlated jumps, we would like to see them not too far one from another, such that they can interact by a force. Of course, we can repeat the same argument for any increase of $u_{\perp}$ from a finite reasonable value $u_{\perp}^{(1)}$ to another finite reasonable value $u_{\perp}^{(2)}$. We have not proved such an argument rigorously for the case 0 is not one of these two values, but we doubt that anyone would claim the opposite to be true. Now what one sees in a speckle experiment at a single value of $Q_{B}+q_{\|}$is exactly a change of intensity (in the form of an exponential decay) between two values close to a main value, that is interpreted to correspond to such a change of amplitude of the "sine wave". This does not correspond to any normal interpretation of the concept of a mode.

\section{Lack of uniqueness}

There exist other modulations than of the phonon type, e.g. compositional modulation, or magnetic modulation. They can be calculated by exactly the same formalism as for a displacive modulation and will yield satellites at the very same positions in reciprocal space. The information is thus unspecific. We cannot claim without further justification that we have a displacement modulation.

When a specific interpretation for an observation is claimed, and when the interpretation is a priori not unique, like it is the case with satellites in a Fourier spectrum, the choice of the specific interpretation must be motivated. It can happen that the motivation given for the claim is not sufficient to make away with all possible ambiguities. This means that the charge of proof for the claim has not been met appropriately and that there are loopholes in the justification. When this is the case, this will in general give rise to objections. A good way to point out that the uniqueness of an interpretation has not been proved, is giving the possibility of a counter example. It is pointless and not appropriate to reply to the suggestion of such possibilities that one would have to prove them, since that would amount to a reversal of the charge of proof. E.g. when one wants to point out a loophole in a proof of a mathematical theorem, this does not imply that one would have to prove that the theorem is wrong. Pointing out a possible loophole shows that the proof is incomplete, and as such that the proof of the theorem is wrong. In an inductive science like physics, there is no parade against infinite scepticism. Therefore, the objections must remain reasonable. All possible reasonable objections must be duly incorporated in the discussion of the interpretation of the data, and it must be clarified how bad they could be. We want to raise the reasonable objection that the interpretation of the diffuse scattering data is in bad lack of proof of uniqueness. The complicated structure of the present paper, with its many subdivisions in sections, subsections, etc... is probably enough to show the profound confusion this has produced.

1. Chemical Disorder. The authors have speculated several times $[6,12]$ about the possibility that chemical disorder could be a kind of phason defect. In reference [6] it is e.g. stated that de Boissieu "wonders if the perp-space Debye-Waller factor component is just another way of accomodating chemical disorder of atoms". It is also stated that "it is in agreement with the diffuse scattering". It is thus obvious that this possibility must be ruled out, before one can claim that the modulation is displacive. As pointed out above, chemical modulation can be treated by exactly the same formalism as displacive modulation. In that case, it is the complicated decoration of the atomic surface that interacts with the "sine wave". The change of the type of atom is then possible by varying the amplitude of the "sine wave", and the variation can occur without necessarily implying a phason jump. In other words, in this case, the "reason" for the change of type of atom is not given by the superspace description in se. We may finally add that in AlMnPd, a strong component of diffuse scattering has been observed at small angles [13], that is flat with $Q$ and has an intensity that corresponds to what one would obtain from a calculation assuming complete chemical disorder. This seems to indicate that the presence of at least some chemical disorder has to be taken as a very serious possibility. Introducing a chemical modulation "sine wave" can be done 
without any reference to elasticity. This implies that when the data are due to chemical disorder, they are irrelevant for the elastic stability issues raised by the random tiling model, as Widom's theory is based on displacive modulation.

2. An alternative type of displacive modulation. But even if we stick to a displacement modulation, there is an alternative. Imagine a QC $\mathbb{Q} \mathbb{C}^{(\alpha)}$ obtained by replacing the atomic surfaces $\mathcal{W}$ of $\mathbb{Q} \mathbb{C}$ by other atomic surfaces $\mathcal{W}^{(\alpha)}$ that are tilted by a small angle $\alpha$ with respect to $\mathcal{W}$, and whose lengths are adjusted such as to keep the condition of conservation of atoms satisfied. 14] The whole derivation of the calculation of the Bragg peaks of $\mathbb{Q} \mathbb{C}^{(\alpha)}$ remains the same. Hence, the positions of the Bragg peaks are the the same in $\mathbb{Q} \mathbb{C}^{(\alpha)}$ and $\mathbb{Q C}$. Only the intensities of the Bragg peaks are changed. The Fourier transform of the tilted atomic surface varies along the direction of this atomic surface, but does not vary along the direction that is perpendicular to it, such that the value of $Q_{\perp}$ of the Bragg peak that occurs in the expressions of the intensities $\sin \left(Q_{\perp} W / 2\right) /\left(Q_{\perp} / 2\right)$, must be replaced by $Q_{\perp} \cos \alpha-Q_{\|} \sin \alpha$. As the length $W$ of the atomic surface also enters the expressions, it must also be properly modified to the new value $W / \cos \alpha$. The final result is $\sin \left[\left(Q_{\perp}-Q_{\|} \tan \alpha\right) \frac{W}{2}\right] /\left[\left(Q_{\perp} \cos \alpha-Q_{\|} \sin \alpha\right) / 2\right]$.

Again we modulate the positions of the undecorated "supercrystal" with a sine wave $u_{\perp} \sin \left(q_{\|} x_{\|}\right)$. Note that this is slightly different from cutting the tilted atomic surfaces with the "sine wave", because in the latter, the value where the amplitude of the "sine wave" must be calculated would be slightly shifted along $E_{\|}$. Call the resulting QC $\mathbb{Q} \mathbb{C}^{(\alpha) *}$. As rigorously the same kind of calculation comes into play for the satellites as for the Bragg peaks, we see that also the satellites of $\mathbb{Q} \mathbb{C}^{(\alpha) *}$ will be in exactly the same positions as in $\mathbb{Q} \mathbb{C}^{*}$ and that only their intensities will be different. Note that, in the end, the factor $\mathbf{Q} \cdot \mathbf{u}_{\perp}$ can only lead to a $\mathbf{Q}_{\perp}$-dependence, due to the scalar product with $\mathbf{u}_{\perp}$. There is a small variation in the intensities of the satellites in that they have now to be calculated as $\sin \left[\left(Q_{\perp}-\left(Q_{\|}+q_{\|}\right) \tan \alpha\right) \frac{W}{2}\right] /\left[\left(Q_{\perp} \cos \alpha-\left(Q_{\|}+q_{\|}\right) \sin \alpha\right) / 2\right]$. As $q_{\|}$is small this will deviate from the value at the Bragg peak by a term of the order $W \alpha q_{\|}$, which is certainly second order with respect to $\left(\mathbf{Q} \cdot \mathbf{u}_{\perp}\right)^{2} / 4$, such that the intensity of the diffuse scattering remains uniquely dependent on this parameter (The real effect of variation of the diffuse scattering intensity with $Q_{\|}$lies in the variation in the intensity of the Bragg peak with which it scales).

Hence, there is no qualitative difference between the diffraction diagrams of $\mathbb{Q} \mathbb{C}^{(\alpha) *}$ and $\mathbb{Q} \mathbb{C}^{*}$. It may disturb at first sight that the modulation produces displacements along $E_{\|}$, but this not conceptually different from the situation with phason jumps. It is thus a possible alternative.

This alternative is more physical. In $\mathbb{Q} \mathbb{C}^{(\alpha) *}$ there will be small atomic displacements in every atom of the QC, even when $u_{\perp}$ is very small. Nobody will find it difficult to believe from such a picture that the atoms are elastically coupled from neighbour to neighbour. There is thus no postulate of spooky correlations over large distances between two isolated jumps, while the whole intermediate region remains unaffected, as in $\mathbb{Q} \mathbb{C}^{*}$. There is no such problem that the closer two jumps are following each other in time, the further away they must be in space. Moreover, when $u_{\perp}$ is very small, these small atomic displacements will outnumber the very scarce atomic jumps.

An essential point is that the real-space atomic diplacements created by the fluctuation of the cut are much larger and not harmonic in the model of the authors. An atomic jump can only occur in an double-well potential, and the function that describes such a potential is at least of the fourth degree. The potential is thus not harmonic. We think that our alternative leads to a less unphysical interpretation of the data, without a real necessity to invoke tile flips as the basic ingredient. The displacement fields are just like classical phonon displacement fields, except for the fact that they are parameterized by other, perpendicular space coordinates. We also want to stress that the procedure of tilting the atomic surfaces is only a first-order approximation to illustrate the idea. In reality, we should introduce a kind of devil's stair case in order to account for far-away changes of configurations. Furthermore, we should modify the atomic surfaces in such a way that it does preserve the symmetry. Duneau [15] has shown that in an admittedly wrong, polynomial approach for icosahedral symmetry, the minimal degree of the polynomials that comply with this condition is three. The idea of modulating the atomic surfaces finds its confirmation in numerical calculations of the dynamics, where it has to be introduced in order to relax the initial system. The idea of modulating the atomic surfaces is also present in a work of Steurer, 16] who calls it the IMS setting (as opposed to the QC setting).

It remains to explain how the diffuse scattering resulting from our alternative model could decrease when the temperature is raised, but there are many possibilities to do this. Widom's instability [17], with tile-flip phason elasticity replaced by a phason elasticity based on small atomic displacements, would already to the job. But it is even not necessary to claim that the QC would not be stable. A mere softening of the elastic constants would do. There are many examples known of elastic constants or phonon modes that soften in a given temperature range, without triggering any phase transition at all.

It is even not necessary to invoke a softening of the elastic constants. It could just be that the system acquires supplementary possibilities to reduce the strain. One possibility is e.g. that thermal vacancies contribute to the relaxation of the observed strain fields. It is quite plausible that their number becomes significant at the temperatures where the fluctuations observed by the authors set in. Also fast phason hopping between two positions could help in relaxing strain fields. 
We may add a note about the fact that the diffuse scattering seems to follow predominantly the phason elastic constants. The diffuse scattering reveals "frozen" phasons rather than "frozen" phonons. Perhaps one thinks this is surprizing, and perhaps one thinks it must be full of highly significant information. But a frozen phonon would stipulate an amplitude for an atomic displacement field in a certain place, just on the basis of its position coordinate, without any hindsight on its environment. The phonon wave could be just totally out of phase with respect to the reality of the local environment. In sharp contrast with this, the phason variable is a parameter that refers to the local environment. In order to know the environment of a point $x$ in a crystal, it suffices to take the non-integer part of $x / a$, where a is the lattice parameter. Hence $x$ is a good quantity to define a phonon displacement field in a crystal, as it contains the necessary information about the local environment. In a QC, $x_{\|}$is not a good parameter to define a displacement field in this respect. The parameter $x_{\perp}$ is much better suited to define the local environment. We think that this indicates that a displacement field in a QC can only reasonably expected to be of the frozen phason type.

3. Other possibilities? We cannot pretend that the list of possible alternatives has been exhausted with these two counter examples. The whole problem with the interpretation of the diffuse scattering is that it is hopelessly difficult, due to the large amount of diffuse scattering scenarios in general, combined with the complexity of the structure and of quasiperiodicity, more specifically. It is for this reason that it is impossible to propose with certainty an alternative interpretation of the diffuse scattering data, and that we were obliged in the previous lines to limit ourselves to suggesting the existence of alternative possibilities. This caution on our behalf should not be misrepresented as a weakness of our viewpoint with respect to the viewpoint that has been published, since that would amount to a reversal of the charge of proof. It rather illustrates the weakness of the published viewpoint in that it has taken a non justified shortcut to the necessary caution. We refer the reader to Section VI where we develop a completely different approach and show how a jump model with a single diffusing tile reproduces several characteristics of the data, while there is no modulation of the superspace lattice whatsoever.

We should also warn the reader against a play of words. As the authors have rightly pointed out, the word "phason" has been used with many different meanings. This was also pointed out by Janssen et al [4] and, much earlier, in reference 18]. Therefore it is all the more surprizing that the authors rely on the mere fact that their data can be described in terms of "phason elasticity" to identify them without any further discussion with phason jumps, as though this would be self-evident. Clearly concepts cannot be identified on the mere basis that they are homonyms. The two examples of chemical disorder and small non-phason-jump small atomic displacements clearly may have physical realizations that could be described by a "wave" with perpendicular-space polarization, even if they both can have also other physical realizations that contain a parallel-space component in the polarization.

\section{A possible ambiguity}

There is not a single $q_{\|}$-value but a whole distribution of them. It is obvious that there is an ambiguity that has not been addressed: The perpendicular-space amplitude $h_{\perp}$ of the ondulation is not unambiguously defined: At each atomic position $h_{\perp}$ can be varied at will as long as it does not lead to a swap of atomic surface. In between the atomic positions $h_{\perp}$ can also be varied. The physical situation in $E_{\|}$allows thus for more than one decomposition in "sinusoidal phason waves", it i.e. in sets $Q_{B}+q_{\|}$. This is perhaps connected to the fact that $Q_{B}+q_{\|}$can also be written as $Q_{B}^{*}+q_{\|}^{*}$, where $Q_{B}^{*}$ is another Bragg peak. The information at $q_{\|}$is thus not unambiguous. It will contain a strong contribution from a strong Bragg peak and other, weaker contributions. We can reduce the importance of the latter problem by making the speckle measurements in the vicinity of a strong Bragg peak $Q_{B}$, such that the decomposition $Q_{B}+q_{\|}$will dominate over all other ones.

\section{How is a non-sinusoidal ondulation of the cut Fourier-decomposed?}

While the Fourier transform of a sine-wave modulation will produce a set of $Q_{B}+q_{\|}$-values and corresponding intensities that allow to reconstruct a field of pure phason jumps, it is not granted that the Fourier decomposition of the whole ondulation of the cut can be kept stepwise, putting each atomic jump as a whole into one set of $Q_{B}+q_{\|}$-values that reconstruct a sine wave that will generate this jump. Two jumps in succession of the same atom, that do not result in a zero displacement, will not forcedly lead to a Fourier decomposition that contains pure phason jumps in the $Q_{B}+q_{\|}$-sets. We see that this raises the question of how large the amplitude of the sine wave can be without ceasing to be sufficiently small for the validity of the approximation that is claimed to justify the formalism. In our development above, we have more or less acted as though the amplitude $u_{\perp}$ of the "sine wave" were less than the amplitude of $\mathcal{W}$, in order to remain within the limit of small amplitudes, and to stay away from problems like violating the criterium of the conservation of the number of atoms. This is not an important restriction, as what we want to deal with in general are small variations of the amplitude, and the only thing that really matters is that the total ondulation of the cut conserves the number of atoms. In any case, it may thus well be that a set of measured 
$Q_{B}+q_{\|}$-values with its intensities defines fractional jumps. What will remain of the interpretation of the diffusion constant analysis in terms of a "sine wave" of jumps if this is the case? The data from $Q_{B}+q_{\|}$-sets may well reflect small atomic displacements that are not whole phason jumps. A field of such small atomic displacements cannot be distinguished from a field where the displacements are not the result of only phason jumps. This underlines once more our argument that it cannot possibly be claimed on the basis of the data, that what one observes is due to disorder induced by tile flips.

\section{G. Summary}

In conclusion of this Section, we have shown that the interpretation of an intensity at $Q_{B}+q_{\|}$in terms of a periodic displacement field with wavelength $2 \pi / q_{\|}$of correlated jumps is wrong and unphysical. It contains several tacit, misleading conjectures, whose truth is just taken for granted, while they are obviously fallacies.

\section{COHERENT DELUSIONS}

\section{A. Important Warning}

Coherent scattering signals collect contributions from all particles of the system that have a non-zero coherent scattering amplitude. It is thus a many-particle signal. Despite all possible folk lore, this should not be overinterpreted. That the scattering is coherent does not prove that it corresponds to a "collective" signal or to a signal of some correlations, as the latter implies that the particles are no longer independent and move in a concerted, correlated fashion, due to some coupling or interaction, as is e.g. the case for phonons. That can very well be the case, but it is not a necessity. The oppositions independent vs. correlated, coherent vs. incoherent, single-particle vs. many-particle cannot be amalgamated.

(1) Coherent scattering with a certain structure can also occur in a system wherein the dynamics of all particles are totally independent. A clear example of this are the Monte Carlo simulations of Tang et al. 10, 11] The tile flips are here completely random and independent, but they lead to a clear coherent signal that is actually very similar in its reciprocal-space properties to the one observed by the authors (It is only the temperature dependence that permits to invalidate the pristine random tiling model used in this simulation). The same conclusions have been reached by Naumis et al.19] They stated that in the long-wavelength limit one cannot draw conclusions as to the presence of correlations, because also totally uncorrelated jumps will give rise to a coherent signal.

(2) Coherent scattering signals can be obtained from the dynamics of a single particle, provided it is a coherent scatterer. This is independent by definition.

(3) Conversely, many-particle systems can give rise to incoherent signals, even if the dynamics are strongly correlated. It just suffices that the particles are incoherent scatterers.

The reader who wants to come to terms with these issues is refered to reference 20. We will discuss how one calculates coherent scattering signals below.

\section{B. Atomic jumps in self-diffusion problems are not correlated}

It is generally admitted that phason jumps can give rise to long-range atomic self-diffusion. The idea has been pushed to the extreme in the model of Kalugin and Katz 21], which predicted extremely fast diffusion, but no evidence has been found in favour of this prediction. What has been observed is extremely fast phason hopping. We think that there is no absolute proof for the possibility that phason jumps lead to diffusion, due to the simultaneous presence of vacancy diffusion. But this is nevertheless generally believed to be true, because it is hard to see an obstacle against it. In any case, Francoual et al. make an analysis of their data in terms of a macroscopic diffusion constant. But in all atomic self-diffusion studies, the prevailing paradigm is that atomic jumps are not correlated over large distances. This adds up to our previous arguments that postulating correlations between atomic jumps over long distances is unphysical.

Such an objection cannot be rebutted by stating that it would be a mere speculation. First of all, the objection just formulates the prevailing paradigm. In this respect it is the postulate of correlations between the jumps that appears as a speculation. Secondly, it would consist in a reversal of the charge of proof.

\section{Atomic jumps in order-disorder transitions}

The objection can also not be rebutted by invoking an order-disorder transition. First of all, invoking an order-disorder transition completely changes the context. It is difficult to see how an order-disorder transition could 
tell us something about atomic jumps in a self-diffusion process. Secondly, atomic jumps do not become correlated in an order-disorder transition. Once again, it is only the atomic positions, not the atomic jumps, that become correlated in an order-disorder transition. What one can e.g. do to illustrate this point in the situation close to the order-disorder phase transition is considering a periodic lattice of asymmetric double-well potentialss. E.g. the minima of the left wells lie much lower than the minima of the right wells. In each double well, an atom is allowed to jump. When the temperature rises the difference between the two wells becomes smaller, and at high temperature the wells are completely symmetric, while at the transition temperature they are highly asymmetric. At all temperatures the jumps in the different double-wells are stochastic, but when the temperature decreases, the jumping atoms will have a much longer residence time in the lower left well minima than in the higher right well minima. While at high temperatures the residence times will be equal, because the two wells are equally deep. The asymmetry will develop when the temperature is decreased. In the asymmetrical double-well the jump rates left-right and right-left are different, and their ratio is governed by a Boltzmann factor. Such models have been used to describe the phenomenology of order-disorder transitions. This clearly shows that the jumps remain independent, while it is the most probable positions of the atoms (within the left well minimum in between the jumps) that become correlated.

\section{Not every reciprocal lattice vector is a wavelength}

In general, there is no guarantee for the attribution of a wavelength to the quantity $q_{\|}$as the authors do. The authors believe that the QC is not stable and that its zero temperature ground state is a crystal. The low-temperature phase is not observed because the kinetics become frozen, such that the phase is not reached. They want to interpret $q_{\|}$as a wavelength associated with the phase transition towards the low-temperature periodic, crystalline phase. It may well be that the random tiling scenario predicts a diffuse scattering as observed by Francoual et al. But the "wave lengths" of this scenario are in superspace, and do not have the real meaning of a wavelength in physical space, as we have already shown. We can also give some physical reasons within the context used by the authors.

As there is no conclusive interpretation of the diffuse scattering, considerations about the nature of the ad hoc stipulated purely hypothetical transition (order-disorder, first or second order) cannot play a role at this stage. Consider thus the clear analogon of a (second-order) antiferromagnetic phase transition.

At approaching the Néel temperature from above, larger and larger antiferromagnetically ordered domains (or clusters) will occur that will take longer and longer times $\tau_{0}$ to decay. This will show up as diffuse scattering intensity centered at the antiferromagnetic Bragg position, e.g. at $\mathbf{Q}=\left[\frac{1}{2}, 0,0\right] \frac{2 \pi}{a}$ of the future low-temperature phase, where $a$ is the lattice parameter of the high-temperature phase. The intensity at $\mathbf{Q}+\mathbf{q}$ will have a characteristic decay time $\tau_{0}$, which is a measure of how long an antiferromagnetically ordered cluster of size $2 \pi / q$ will persist in time without being disrupted by the spin flip dynamics. We see that it is $2 \pi / Q$ rather than $2 \pi / q$ that characterizes the wavelength $2 a$ of the spin wave that is being built up. The quantity $2 \pi / q$ is not a long wavelength of some spin wave, but an instantaneous domain size, a coherence length of the short wavelength spin wave. The time $\tau_{0}$ is not characteristic of the spin flips themselves (which are local), but of the absence of spin flips within a domain of size $2 \pi / q$. These domain sizes increase when the spin flip dynamics slow down on approaching $T_{N}$.

In this discussion, we use the phase transition only to illustrate a possibility of an interpretation. In the context outlined above, this possibility will remain valid in its general ideas, even if there is no phase transition at stake at all: $q$ refers to a domain size, rather than to a wavelength. By analogy, we see that it does not go without justification in the quasicrystal, to associate $2 \pi / q_{\|}$with some hypothetical long wavelength phason wave. Moreover, if there were some wavelength $\lambda$ in the phason dynamics, diffuse scattering should eventually build maxima at new Bragg peak positions at $\mathbf{Q}$ with $Q=2 \pi / \lambda$, or at satellites positions at $\mathbf{Q}=\mathbf{Q}_{\mathbf{B}}+\mathbf{q}$ rather than remaining smeared out over a continuum of positions $\mathbf{Q}_{\mathbf{B}}+\mathbf{q}$ in a distribution centered on $\mathbf{Q}_{\mathbf{B}}$, with $q=2 \pi / \lambda$ (if it is the signature of the mechanism behind the transition). As the diffuse scattering in QCs remains centered at the Bragg peaks of the high temperature regime and its maxima do not define new Bragg or satellite positions, the ad hoc interpretation in terms of critical scattering announcing a phase transition that would not be reached due to the slowing down of the phason dynamics, lacks proof. Note that the slowing down of the spin flips is what triggers the antiferromagnetic phase transition rather than impeding it!

\section{E. Experimental data never contain direct information about correlations between atomic jumps}

We have seen above that the whole discussion about correlations between the jumps originates in a confusion that has been made between correlations between atomic positions and correlations between atomic jumps. We will see below that this confusion has been amplified by overinterpreting the coherence of the signal. The confusion manifests itself already in the apparent paradox that $\lambda$ is not a period of the displacement field, while $2 \pi / \lambda$ occurs in the Fourier spectrum of the disordered quasicrystal. Correlations between atomic jumps can just not be directly observed in an experiment. We would like to stress this point further on the basis of the Van Hove formalism for coherent neutron 
scattering.

The Van Hove formalism says that what you measure in coherent neutron scattering is the Fourier transform of the probability of having a particle $j$ at position $x$ at time $t$ if the same or another particle $k$ (whereby $k=j$ is thus allowed) was present at position 0 at time 0 . Hence, what we deal with in a measurement are correlations between positions and not correlations between jumps. The very same formalism applies, mutatis mutandis, for $\mathrm{X}$ ray intensities.

\section{F. Conflicting time scales}

We have seen that a Fourier component at a single value of $Q_{B}+q_{\|}$corresponds to a density wave for the disordered $\mathrm{QC}$, and that a set of them with the same value of $q_{\|}$would define a displacement field that is not periodic. The time constant for the relaxation of this field is claimed to be of the order of minutes, on the basis of the speckle experiments. Let us first of all point out that it is difficult to claim that two phason jumps separated by a long distance, could by correlated in time at such a time scale of the order of minutes. In the mean time, the corresponding atoms will have jumped independently a huge number (at least of the order $10^{12}$ ) of times, as the relaxation time for individual jumps measured by neutron scattering and Mössbauer spectroscopy lies in the range from a few picoseconds to a few nanoseconds. What permits us to say that phason jump number $n$ at a given position $x$ will be the one phason flip that correlates through the "sine wave" with a flip, at position $x^{\prime}$, hundreds of Angstroms away, a few minutes earlier? Of course it can be argued that one has to filter out the fast components. It has been stated $[\underline{6}]$ that "In this case of the long-wavelength phason fluctuation, individual phason flips, which are local rearrangements of underlying tiles or atomic jumps, correlate at long distance because the same Fourier mode is responsible for them". We would like to point out that this contains a kind of reversal of the causality that is by no means warranted by the information content of the data.

To point out why, it is sufficient to refer to the pristine random tiling model Monte Carlo calculations by Tang et al. 10, 11] This model will yield the very same type of diffuse scattering around the Bragg peaks, as in a model wherein the kind of correlation evoked above is stipulated. This point was also made by Naumis et al. [19] (see above). And this happens despite the fact that all the jumps are totally uncorrelated by definition: What one does in the model is to flip tiles at random by Monte Carlo. The only experimentally detectable qualitative difference between the model simulated by Tang et al. [10, 11] and Widom's model[17] is the T dependence (which is opposite). The model of Tang et al. 10, 11] will yield similar time decays of the speckle, because any wavelength that might have been built up in the fluctuation pattern is entirely random and must therefore decay away again with time. Of course, these chance Fourier components are also building up with time following the very same time constants. They come and go. In the model of Tang et al., 10, 11] there is no correlation whatsoever between the jumps, but of course the random configurations of the system will yield the Fourier components with their time decay. These are chance Fourier components of random correlations. The authors invert the logical order of cause and effect by attributing the origin of the correlation to this Fourier component, such that it would be the Fourier component that causes the fluctuations. In reality the fluctuations are random, and the Fourier analysis of these fluctuations yields the chance Fourier component. There is not the slightest element in the experimental data that permits to justify this kind of reversal of cause and effect that is present in the claims of the authors. Such a reversal might be necessary to make the conceptual change from the model of Tang et al. 10, 11] to a reading of Widom's model 17] in terms of tile flips (rather than small atomic displacements), but there is nothing in the experimental data that can be claimed to be evidence for it.

Certainly, in the model of Tang et al. 10, 11] it can occur that locally, a tile flip only becomes possible after another one, and one could build a chain of such possibilities over a long distance. But many other tile flips disrupt this chain all the time at a very high rate, as explained above. The origin of the coherence of the signal is not the presence of such marginal chains. What is overinterpreted in the data is the fact that the coherence of the signal is not due to correlations between atomic jumps, but to correlations between atomic positions. In fact, the totally uncorrelated jumps in this model will nevertheless give rise to strongly structured coherent signals, but these are due to the correlations between the atomic positions. The paradox is thus, once gain, due to a confusion between correlations of atomic positions and correlations of jumps.

Altough it is not relevant for the data, its is perhaps interesting to end this subsection on a remark about random correlations between jumps. For the atomic jumps themselves one should not confuse the constraints of the random tiling model with some correlations or lack of independence in the tile flips. The jumps are totally uncorrelated within the given set of constraints dictated by the random tiling model. To give an analogon: For two completely independent walkers in a city where all streets run only eighter North-South or East-West, like New York (without Broadway), we might find a mysterious correlation in that they are found to walk always only in mutually perpendicular or parallel directions. This is not a mysterious correlation between the two independent walkers, but a constraint imposed by the city map of New York. Hence, yes it is true that the directions of the jumps in the random tiling model are 
strongly "correlated", but nevertheless the jumps are totally independent by the very construction of the Monte Carlo simulation.

\section{THE TEMPERATURE DEPENDENCE}

\section{A. What it is all about}

The diffuse scattering data cannot correspond to disorder generated by phason jumps. Up to now we have analyzed the diffuse scattering in the assumption that it would be due to tile flip disorder. We have already pointed out alternative possibilities, e.g. chemical disorder. Here we show that the data simply cannot correspond to quasicrystal disorder that would be created by tile flips.

The quasielastic neutron scattering signal that corresponds to tile flips has been studied in great detail. The intensities of the quasielastic signals in these data show that the number of phason jumps increases when the temperature is raised. This is model-independent factual information. If the diffuse scattering observed by the authors were to correspond to tile flip kinetics it should thus follow the same temperature behaviour as in the neutron data. In reality it follows the opposite one: the diffuse scattering diminishes when the temperature is raised. This contradiction is unassailable. Perhaps, this requires a more detailed discussion.

\section{B. Coherent and incoherent scattering in many-particle problems}

\section{Method of Calculation}

First of all the reader should check in Reference 20] how a coherent quasielastic signal is calculated. Such a coherent quasielastic signal is the temporal Fourier transform of the speckle signal whose intensity decays exponentially with time. We cannot possibly reproduce the whole development of that paper. Perhaps it suffices to say that one first has to map out the whole set of configurations $\mathcal{C}_{\mu}$ that the system can take. Each configuration $\mathcal{C}_{\mu}$ can be represented as a point in a higher-dimensional space. When an atomic jump with relaxation time $\tau_{0}$ takes the system from configuration $\mathcal{C}_{1}$ to configuration $\mathcal{C}_{2}$, we connect the corresponding points by a line that we label with $\tau_{0}$. This way the whole dynamics can be mapped in the terms of a graph in higher-dimensional space. We can then say that the whole system is an abstract, single particle that diffuses through first-neighbour jumps on the higher-dimensional graph. This high-dimensional diffusion problem can then be expressed in terms of a set of coupled linear differential equations with a jump matrix, just as the normal, less abstract diffusion problem of a single particle in physical space.

We may note that not only coherent scattering, but also incoherent scattering for a many-particle system can be calculated following this method. Contrary to folk lore, this leads to a more complicated and structured neutron signal than coherent scattering. In coherent scattering, it is not relevant where the individual particles are within a configuration. All configurations obtained by particle exchange operations are in the same equivalence class. All that counts in coherent scattering is the configuration. But, in incoherent scattering one must keep track of a single particle. In incoherent scattering, two identical configurations, where an individual particle finds itself in different positions, are therefore no longer equivalent. The configuration space to be considered is therefore much larger in the case of incoherent scattering. The reader can verify this on the calculation of the model in reference 20].

There is still one step missing if we want to compare this with the speckle data, which are obtained with X-rays. In general the scattering formalisms for various techniques are quite analogous. They can be just transcribed mutatis mutandis from one probing particle to another one. But it is only with the advent of synchrotron sources that the study of dynamics has been opened to X-ray studies. In fact, X rays do not have the necessary energy resolution (which lies at the very best in the $e V$ range) to separate even the broadest quasielastic scattering (which occurs in the $\mu e V$ or meV range) from purely elastic scattering. The diffuse scattering observed is thus an energy-integrated whole of all quasielastic and elastic signals. That means that one has to add up all quasielastic form factors from the calculation described above in order to obtain the diffuse scattering signal. A diffuse signal at a paricular $Q$-value will in general arise from many contributions with different relaxation times. But in the case of the speckle data, there is a time dependence, such that the separation between the quasielastic scattering and the elastic scattering is obtained in the time domain. It also appears that, at least in a first approximation, at a given $Q$-value, one measures only one, dominant value of the many relaxation times that are probably present.

\section{Applying it to quasicrystals}

If one were able to diagonalize the huge jump matrix that describes the whole of the phason dynamics, along the methods of calculation descibed above, one would find a (very large) number of characteristic times, each leading to a Lorentzian signal with a dynamical structure factor. Such a jump matrix exists both for the coherent and for the 
incoherent scattering case. There is a coherent and an incoherent signal even for the case of completely independent jumps. Such a theoretical treatment is beyond reach however, due to the sheer size and complexity of configuration space. The observed neutron scattering data correspond to the sum of coherent and incoherent scattering (contrary to the statements of reference [1] that the neutron scattering signals would be incoherent). The long-time signals attributed to phason dynamics in reference [1] would just correspond to some of these Lorentzians, with very long relaxation times.

These long relaxation times are not elementary parameters of the jump model, but functions of more elementary jump times. These functions pop up as the inverses of the eigenvalues $\lambda_{j}=f_{j}\left(\tau_{1}, \cdots \tau_{n}\right)$ of the jump matrix that has been defined in terms of the few more elementary jump times, $\tau_{1}, \cdots \tau_{n}$, which are much faster. The Q-dependence of the intensities of all Lorentzians $\Lambda_{j}$ is given by the corresponding structure factors. Any temperature dependence enters into the model through the temperature dependence of the elementary jump times in terms of activation energies. Through the functional dependence $\lambda_{j}=f_{j}\left(\tau_{1}, \cdots \tau_{n}\right)$ evoked, the temperature dependence of all Lorentzians is thus dictated by the temperature dependence of the elementary jump times. According to crude criteria such as increasing or decreasing of jump times or intensities, the long time dynamics should thus have the same temperature dependence as the short time dynamics, and any person who wants to formulate a claim that they could show opposite behaviour will have to work very hard to gain credibility for it.

But the experimental observation (from quasi-elastic neutron scattering) that the intensity, rather than the width of the fast signals increases with temperature is unusual. In a first approach, one might argue that it could be due to the finite energy resolution of the neutron scattering experiments: At low temperatures the dynamics are too slow to be resolved and appear as elastic. At higher temperatures they become resolved leading to the illusion that the intensity of the elastic peak decreases and the intensity of the quasielastic signals increases accordingly. But this is not what happens: The width of the quasielastic signals cannot be detected to change with temperature, while their intensities change drastically in a way that cannot be attributed to some broadening. One needs to introduce an assistance scanario to explain this very unusual behaviour.

But what one observes in the diffuse scattering and has been called the effect of an inverse Debye-Waller factor, is that the intensities (i.e. structure factors) of the very slow signals decrease while the temperature is raised, with the intensities being transfered to the Bragg peaks! If there were no assistance scenario in the jump dynamics, the intensity and its Q-dependences for a given Lorentzian $\Lambda_{j}$ would remain the same at all temperatures. In fact, the structure factor of the Lorentzian $\Lambda_{j}$ associated with $\lambda_{j}$ is not changed by a speeding up of the dynamics (allowance made for the phonon Debye-waller factor). At the very best, its intensity at a given Q-value would appear to be associated with a faster relaxation time, through the functional relationship $\lambda_{j}=f_{j}\left(\tau_{1}, \cdots \tau_{n}\right)$. If one wants to change $f_{j}$ or the structure factors, rather than just $\tau_{1}, \cdots \tau_{n}$, one has to introduce special assumptions. The observation that the quasielastic neutron scattering intensity increases with temperature forced us to introduce such an assumption in the form of an assistance scenario, as even the elastic intensity is determined by the jump model: It corresponds to the eigenvalue 0 of the jump matrix, and hence its intensity or structure factor should normally not change with temperature. In other words: Allowing for the effect of the Debye-Waller factor due to the phonons, the ratios of the various structure factors, including the elastic one, should have remained the same. In the assistance scenario, the elastic intensity decreases with temperature, because one introduces long-lived excited states, whose population is governed by a Boltzmann factor with a large activation energy. What kind of most extraordinary ad hoc assumptions would have to be introduced into the jump model in order to obtain a decreasing diffuse intensity as observed by the authors, that could be attributed to phason jumps despite the fact that the quasielastic intensity corresponding to fast phason jumps has been observed to increase by neutron scattering?

To resume the situation: The number of tiles that flip increases when the temperature is raised. Therefore, the intensity of the signal that should betray the presence of these tile flips, e.g. off-Bragg-peak diffuse scattering claimed to correspond to the structural disorder produced by the flips, should also increase when the temperature is raised. Such a temperature dependence runs contrary to what the authors observed.

The authors [1] then decided to proceed by adopting an "alternative random tiling model", proposed by Widom, wherein the diffuse scattering intensity decreases when the temperature is raised. This is certainly important for comparing different versions of the random tiling model, that differ in their predictions about the temperature dependence of the data, but it sidetracks the attention with respect to the important issue we want to address here: Such a change of model does not change a iota to the fact that the diffuse scattering intensity cannot be attributed to structural disorder produced by tile flips as it has the wrong temperature behaviour. It follows that the diffuse scattering, which they call the "phason fluctuations" of the alternative random tiling model, must be dissociated from tiling disorder, i.e. the "phason fluctuations" are not tile flip kinetics.

This point cannot be rebutted by arguing that this would not be contradictory because it is only the pristine random tiling model, as used in the simulations of Tang et al. 10, 11 that would ask for an intensity that increases with rising temperature, while Widom's more elaborate model 17 can also incorporate other temperature dependences. That would be a totally pointless misrepresentation of the issues. We are not opposing the temperature dependence 
of the data to the predictions of the random tiling model (although it is interesting to point out that the temperature dependence discards the pristine model in favour of Widom's model[17]) we are opposing the temperature dependence to the information given by quasielastic neutron scattering data!

\section{WHAT IS PHASON ELASTICITY ON THE MICROSCOPIC LEVEL?}

By formulating their claims in a macroscopic language of phason elasticity, the authors [1] created a language barrier: Nobody understands how the macroscopic phason elasticity is supposed to relate to the microscopic-level tile flips, if it does at all. And therefore, nobody knows how the previously mentioned microscopic objections about the temperature dependence should be translated across this language barrier. In Widom's paper 17 it is stated that on lowering the temperature the QC moves away from the ideal random tiling conditions and that this drives an elastic instability. It is not told how this should be described on the microscopic level. The elastic instability could e.g. correspond to a distortion of the tiles rather than to their mere flips. Widom's paper 17] talks about an inverse Debye-Waller effect on the elastic intensity.

\section{A. Debye-Waller factors}

What the authors call the inverse Debye-Waller effect is the fact that when the temperature increases the diffuse scattering decreases, and the intensity is transfered to the Bragg peak, such that simultaneously the intensity of the Bragg peak increases.

A true Debye-Waller diminishes all the elastic intensity at the profit of the inelastic intensity. There is a sum rule between the elastic and the inelastic intensity. The Bragg peaks and the diffuse scattering intensities are both elastic intensities, such that they should be both affected the same way. What is the solution of this apparent contradiction?

What Widom calls the Debye-Waller factor in his paper 17] is not the true Debye-Waller factor, but a theoretical auxiliary quantity that consists on integrating on only the very long-wavelength modes (i.e. on only an infinitesimal domain of $Q$-vectors). It thus excludes the whole phonon density of states (as e.g. measured by Suck), except that infinitesimal part. Similarly, it excludes all the phason dynamics measured by TOF neutron-scattering, except an infinitesimal part. Within this long-wavelenth approximation Widom then calculates his auxiliary quantity that indeed applies to the transfer of intensity between the Bragg peak which is elastic and the diffuse scattering which becomes inelastic in this approach. This leads to the interesting possibility that the true Debye-Waller factor, obtained by including the rest of the full $Q$-range, could again invert the tendencies described by Widom, 17] i.e. some Bragg peaks could show the inverse effect, while others could show the normal effect.

\section{B. Diffusion constants}

We may note that a linear relationship $\tau_{0} \propto 1 / q^{2}$ certainly does not prove that the speckle data are produced by a diffusion mechanism. The relevant parameter for the diffuse scattering is $1 / q^{2}$, such that the first terms of any Taylor expansion for the underlying physics will lead to $\tau_{0}=C_{1}+C_{2}\left(1 / q^{2}\right)$. The authors have previously considered the relationship $\tau_{0}=C_{1}+C_{2}\left(1 / q^{2}\right)$ and this resulted in a much better fit of their data. Such a relationship is totally unspecific. In general, fits of the type $\Gamma=1 / \tau_{0}=\Gamma_{0}+D q^{2}$ (where $\Gamma_{0} \neq 0$ is an indication for confined motion) are used to analyze data when we already know that they are produced by a diffusion mechanism, not to present the data as supplementary evidence that a diffusion mechanism would be at work.

But let us admit that the analysis of the authors in terms of a diffusion constant is correct. We want to point out that the use that the authors make of Lubensky's statement $\underline{\underline{3}}$ ] that "phason modes are diffusive" is misleading, as it creates the impression that the data analysis would contain proof for their interpretations, while this is not true.

E.g. Huang scattering in standard crystals is traditionally described as a "frozen phonon". (This has nothing to do with phonon dynamics: The kinetics of frozen phonons will lead to (very narrow) quasielastic scattering, while dynamical phonons correspond in general to non-zero frequencies). The kinetics of frozen phonons shall be "diffusive" in many instances, although phonons are not qualified as diffusive by Lubensky. [3] That the kinetics of the diffuse scattering is of the relaxational or even of the "diffusive" type, in the interpretation Francoual et al. want to give to it is thus unspecific.

There is a conceptual contradiction between wanting to describe a phenomenon as diffusive and taking simultaneously a "sine wave" Ansatz as the authors do. The "sine wave" makes a decay that occurs simultaneously over the whole of space, while a diffusive phenomenon should progressively spread out in space. Diffusive "sine waves" are a contradictio in terminis (see also below).

We may finally note that time scales of the order of minutes put the interpretation of the data beyond any possible cross-checking with other spectroscopic techniques. This is a dangerous situation that demands for extreme caution. It is preferable to have a situation where we can follow the physics from time window to time window, with different 
techniques that have overlapping time scales if we want to link two observations in vastly different time domains to a same phenomenon as the authors do, by identifying diffuse scattering on the minute time scale with phason dynamics on the pico-second time scale.

\section{Phason elasticity}

It might be misleading that theoretical physicists also use the term elasticity for situations where there are no interactions between atoms. E.g. in the simulations of Tang et al., 10, 11 the tile flips are totally independent, but still a "phason elasticity" can be defined to refer to an "entropic restoring force". If this is not appreciated properly, it can lead to more confusion in terms of "elastic wave" pictures that do not apply.

\section{DELUSIONS ABOUT CORRELATIONS}

\section{A. Introduction}

\section{Conficting paradigms}

The "phason wave" is claimed to be collective and diffusive, to consist of correlated jumps and to have a sine or cosine profile. There are serious problems with the physics of this claim:

A first, and major problem is that nothing is done to clearly explain to the reader what this is supposed to mean, e.g. by an unambiguous definition or by a detailed description in terms of a number of figures showing the diplacement field and how the diffusion process is supposed to proceed step by step on a QC tiling. Instead of that, the claim is put forward as though its contents would be conceptually trivial and self-evident. But this is not at all the case, although it does not transpire on a first reading. It is only when one tries to make sense of the claims, that one gradually discovers the problems, and ends up being trapped within the frustrating task of trying to figure out a reasonable mental representation of what is going on.

What the authors propose is not an explanation but a collection of ad hoc assumptions, which are conceptually contradictory while taken from two conflicting incompatible paradigms, viz. the phonon model and the jump diffusion model.

Diffusion and jump dynamics. In a diffusion process or in a jump model particles are seen to move backwards and forwards stochastically. With time the positions of the diffusing particle become more and more remote from the starting position. After long times, the distance from the initial position is seen to be proportional with the square root of the elapsed time, and the displacement vector has an isotropic distribution. The diffusing particles all move independently: There is no such thing as coherent diffusion at $900 \mathrm{~K}$ in a metallic alloy. A priori, the only correlation that does exist between the diffusing particles, is that two of them cannot occupy a same lattice site simultaneously. In some cases, there can be a local correlation between atomic jumps, in the sense that the jump of one particle can be necessary in order to enable the jump of another particle. We have acknowledged for this possibility already in our very first papers. The diffusion equation contains the first partial time derivative of a site-occupation probability field. That the Lorentzian width in energy of the quasielastic signal is given by $\Delta \hbar \omega=D q^{2}$ is a result that can be derived for incoherent scattering in the case of single-particle diffusion. And then $q$ does not refer to a distance to some nearby Bragg peak. It is a unique single q-value to be taken with respect to $Q=0$. That this result could be extrapolated to the problem of several diffusing particles is a guess, that needs to be proved. Even for the case of the diffusion of two particles on a periodic lattice, the mathematically rigorous calculation is very difficult, because the "fermionic" condition that two particles cannot be on the same site breaks the translational invariance that allows one to diagonalize the jump matrix in the single-particle problem. Of course one can take the approximation that with only two particles the concentration is so diluted that one can neglect without any practical incidence the probability that the particles become so close that one would have to take care of the "fermionic" condition. But it is not obvious at all that the general phason problem on the Fibonacci chain can be treated within such a limit of infinite dilution, as all particles are allowed to jump. It must also be stressed that in the single-particle problem there are no replicas of the intensity around every Bragg peak.

Phonons and lattice dynamics. In a phonon problem there is no stochastic back and forwards wandering process of the particles. The phonon propagates linearly and deterministically on a straight line in a well defined direction with a well determined wave vector. The wave equation contains the second partial time derivative of a displacement field. The position in energy of the inelastic signal is defined for a set of $\mathbf{Q}+\mathbf{q}$-values at a distance $\mathbf{q}$ from the Bragg peaks $\mathbf{Q}$, i.e. the spectrum contains replicas around every Bragg peak.

We may note that in their simplest formulation on a periodic lattice, both dynamical models lead to a set of coupled partial differential equations, that can be expressed in matrix form, and that (e.g. when only first-neighbour 
interactions or jumps to first-neighbour sites are considered) these matrices are identical apart from a pre-factor. Therefore they lead to identical wavelike eigenvectors, due to the translational invariance of the periodic lattice (This is just Bloch's theorem). But this is not the end of the story as these eigenvectors have to be fed into two very different formalisms. The fact that the time derivative is of the first order in the diffusion problem, while it is of the second order in the phonon problem, leads to an essential difference: In the phonon problem we end up with oscillating time behaviour and propagating waves. In the diffusion problem there are no waves: The dynamics of the diffusion problem lead to relaxation with an exponentially decaying time behaviour. The eigenvectors couple also to different formalisms for the calculation of the $Q$-dependence of the signals. One of the important consequences of this is that the phonon problem leads to a $Q$-dependence with replicas of the inelastic signal at every Bragg peak, while the diffusion problem is a priori exepected to be devoid of such replicas, despite the fact that the eigenvectors of the two matrix formulations are the same.

Already at this stage it transpires that phononlike wave behaviour is not what one would call the hallmark of diffusion. There is therefore no way that it could be serious practice to introduce a wave Ansatz for a diffusion process, without any discussion or justification. Despite these very elementary basic principles, the authors cherrypick in each of the two conflicting paradigms those aspects that can be claimed to confirm their views, and ignore the ones that manifestly contradict them. The authors do not touch upon the conflicting nature of the two paradigms, and shortcut the obligation to prove that such assumptions are not mutually exclusive, by showing that they can be derived from a single contradiction-free model. Of course, the isolated components of these claims, taken one by one, are physically sound, such that there is a kind of "déjà vu" that surrounds them and makes them look plausible on a first superficial impression. But the dangerous other side of the medal is that such a methodology can be like assembling griffins, unicorns, sphinxes, centaurs, cyclopes or flying horses from parts of existing animals. E.g. polarization vectors are only defined for propagating waves, not for diffusion phenomena, and equations for atomic jumps are not derived from elasticity considerations.

Statics vs. dynamics. The claims also blend concepts into the dynamics that are static rather than dynamical. The alleged dynamical phason wave is a "sine wave" that in reality has only meaning in a static, structural sense. The calculations presented are calculations of a (component of a) static structure factor. It is meaningless to think of dynamics being accurately described by multiplying a static structure factor with a time dependence in terms of an exponential decay. A dynamical signal is not governed by an expression of the type $S(\mathbf{Q}) e^{-t / \tau_{0}}$, where $S(\mathbf{Q})$ is an elastic structure factor. The $Q$-dependence of a dynamical signal follows a dynamical structure factor. The fact that a polarization vector along perpendicular space is attributed to this purely static description very suggestively creates the impression that it is a dynamical concept, which it is not. The fact that the dynamical phason wave is presented as having a polarization along perpendicular space also clearly shows that the dynamical concept that one wants to introduce is being thought of as being obtained from a cut through higher-dimensional dynamics, beit that this is easily denied, by drawing one's attention to the static presentation. It is very hard to spot that the latter is concerned with a purely static object that does not correspond to dynamics. But the calculation takes exception with the established prescription for the calculation of a dynamical structure factor. We came across the consequences hereof already in Section I, as they reveal themselves through the fact that the time decay of the sine wave introduces completely unphysical correlations over arbitrarily large distances, that are alien to real diffusive phenomena (see I.F.1). In fact, the Darstellung of a static "sine wave" that exponentially fades away from the structure has nothing to do with a dynamical wave, neither of the propagating nor of the diffusive type.

A static structure factor is built up from weighted expressions of the type $\mathcal{F}(\mathcal{C}) \mathcal{F}^{*}(\mathcal{C})$, where $\mathcal{C}$ is a configuration of the system. A dynamical structure factor is built up from weighted expressions of the type $\mathcal{F}\left(\mathcal{C}_{2}\right) \mathcal{F}^{*}\left(\mathcal{C}_{1}\right)$, where $\mathcal{C}_{1}$ and $\mathcal{C}_{2}$ are different (initial and final) configurations of the system. A process wherein the initial and the final state are identical, as in $\mathcal{F}(\mathcal{C}) \mathcal{F}^{*}(\mathcal{C})$, does - by definition - not correspond to dynamics. (Of course it could be argued that there could be a constant $\alpha \neq 1$ such that $\mathcal{F}\left(\mathcal{C}_{2}\right)=\alpha \mathcal{F}\left(\mathcal{C}_{1}\right)$, resulting in a structure factor that is only apparently static. Perhaps this looks as a convincing paradigm. But as we already discussed the thereby postulated long-distance correlations are unphysical. How compelling the evidence is for an interpretation in terms of such a paradigm will be discussed in more detail in subsubsection VI.6.).

We will discuss a first series of models of many-particle diffusion where it can be shown rigorously that there is no wavelike spatial dependence of the signal. It can be shown that for sufficiently small amplitudes, the sine wave proposed is incompatible with any long-range diffusion (see below). This is reminiscent of the threshold in the KatzKalugin scenario (which was however derived under very different assumptions). It is generally believed that in the temperature range of the data of the authors, the Katz-Kalugin percolation transition has not yet occurred. The claim of the authors of a diffusive phason wave is formulated without any mention of this kind of limitation, which clearly reveals the misleading character of its presentation that looks totally devoid of any possible problem. Moreover, for any kind of deformation of the cut that is bounded by this amplitude, the Q-dependence of the signal is smooth (see below), while the sine wave paradigm predicts discrete contributions at positions $\mathbf{Q}+\mathbf{q}$, again without any mention of a possible limitation. 
We will also discuss a model of many-particle diffusion that leads to an intensity pattern that shares four basic features with the diffuse scattering, viz. (1) there is a replica at every Bragg peak, (2) the intensity scales with the intensity of the Bragg peak, (3) it falls of as $1 / q^{2}$ where $q$ is the distance from the Bragg peak, and (4) the relaxation has a time decay constant that is proportional to $1 / D q^{2}$. (It is probably not true that the intensity could be modeled within the framework of the theory of Jaric and Nelsson as being purely due to phason elasticity only, but this can hardly be a point as it is totally obvious that we are dealing here with phason dynamics. Perhaps, we should learn from this that "phason elasticity" is not the 1-1 criterium to decide if a signal is due to phasons that it is claimed to be). But in this model the QC configurations that produce this intensity pattern do not at all correspond to a sine wave ondulation of the cut, and $q$ has no meaning of a wavelength whatsoever. In fact, in this model the set of QC configurations that comes into play is exactly the same one for every $q$-value! This shows the arbitrariness of associating a wavelength with the quantity $q$ and of imposing the modulation wave picture on the data. This picture has thrived on the basis of the difficulty of the calculations that are needed to question its validity.

\section{Problems with elasticity}

A second kind of difficulties that are passed under silence concerns the generalisation of the elasticity theory to superspace. It is true that shifts of the cut can lead to configurations that have very similar energies. However, there is no low-energy path that connects these configurations: The energy levels in the two wells of the double-well potential may indeed have very close values, but they are separated by a huge potential wall in between them, at least a few $\mathrm{eV}$. In other words, as we already pointed out in the previous subsubsection, extrapolating a sine wave concept from a context of thermodynamics to a concept of dynamics is not justified. The energy scale of dynamics is in the $\mathrm{meV}$ or sub-meV region, not in the eV region. In fact, the neutron data suggest that e.g. a vacancy has to come along to assist the jump process (The assistance energy has been measured to be $0.6 \mathrm{eV}$ in $\mathrm{AlCuFe}$ ). It is absurd to postulate the existence of a whole choreography of vacancies (or whatever other assisting processes) as would be needed to produce the long-wavelength correlations between jumps that are suggested by the authors.

It may be reminded at this point that elasticity only plays a second-stage rôle in the calculation of the static structure factor. In a first stage calculating the static structure factor is concerned with a matter of density waves. These waves are parametrized through the six-dimensional Fourier variables $q_{j}$. The diffuse scattering profile must to first approximation be a second-degree polynomial in the variables $q_{j} / q^{2}$. To account for the symmetry constraints it is normal to analyse these intensities in terms of the symmetry-adapted second-rank tensors. The second-degree polynomials follow from symmetry even without calling upon elasticity considerations. Of course, the intensity profiles will comply with these symmetry considerations. Elasticity comes in when we want to take into account the proper weighting of the contributions by the Boltzmann factors, for which an estimate of the energies involved is needed. This elasticity argument is thermodynamical, not dynamical. Hence what the diffuse-scattering formalism calculates are not dynamical waves (with a dynamical structure factor), but a Fourier decomposition in (physically meaningless) density waves of static modulation fields (with static structure factors), which are being thermodynamically weighted by calling in elasticity considerations to estimate the relevant energies. The fluctuation of a "wave" (or more precisely of a Fourier component, see subsubsection VI.6 below) is not the same thing as a wave of fluctuation: That the thermodynamics fluctuate (and that the diffuse scattering allows for a Fourier decomposition) is by no means a sufficient ground for postulating the existence of dynamical waves with a dispersion relation, and a calculation of these fluctuations in terms of a static structure factor as $S(\mathbf{Q}) e^{-t / \tau_{0}}$ does not correspond to an accepted formalism for a calculation of the dynamics.

Obviously other mechanisms for diffuse scattering will be subject to the same symmetry considerations. What will distinguish an interpretation of the data based on such mechanisms from an elastic interpretation will be the values of the weighting factors of the various components. These weighting factors are elastic constants in the elastic interpretation, but have a different meaning in another interpretation. A weighting factor that is different from its value calculated on the basis of an elasticity Ansatz may indicate that the origin of the diffuse scattering is not elastic. It is then not appropriate to call such weighting factors elastic constants. (If an instability is observed, it is then also not elastic. E.g. if the QC needs a very fine tuning of its chemical composition in order to be stable, a small off-stoichiometry of the sample may induce a small chemical instability. One could pass this way through the vicinity of a chemical instability within the phase diagram by varying the temperature, as was suggested by Gruschko. Such possibilities cannot be interpreted within the conceptual framework of a softening of the elastic constants).

The concerns about high energy barriers suggest that we might be in a regime of pinned phason dynamics, where the hydrodynamical theory does not apply, as discussed by Lubensky himself. In fact the whole present approach of the diffuse scattering in terms of a theory of linear phason elasticity hinges on the assumption that we are not in the regime where phasons are pinned. The deep problem that this assumption is not at all proved, is systematically being ignored and eclipsed behind a triumphant presentation of the claims. The fact that phason jumps are observed and that they require a high assistance energy is a strong indication that the QC is still in the pinned regime. It 
was in fact mentioned as a possibility by Lubensky that pinning only disappears at the melting temperature. If Lubensky's approach is complete, it should be able to explain why phasons are propagating and not diffusive in an incommensurately modulated structure like biphenyl while they are diffusive in a QC. In both cases the ingredients are Landau theory and a broken symmetry from a higher-dimensional description. The difference is of course that the atomic surfaces are not continuous in QCs, but this is not an ingredient that enters into the continuum theory.

The picture of an elastic wave with a polarisation along perpendicular space misleads in that the displacements it really brings about are along parallel space, not along perpendicular space. Let us consider an atomic surface that is just about to induce a jump. This atomic surface is centered on a node of the superspace lattice. When a superspace phonon jiggles the nodes of the superspace lattice along the direction of perpendicular space, one gets the impression that the nodes just can make tiny very low-energy vibrations along perpendicular space in a local harmonic potential well, while in reality the real motion induced is a large jump over the barrier, that requires a large assistance energy. In stead of making small displacements within a minimum of a potential well within the harmonic regime, the jumping atom has to go over the maximum of the potential in a highly non-harmonic regime! In other words, it is not correct to treat the displacements of the atomic surfaces as equivalent to displacements of the nodes of the same amplitude, although nothing transpires about this in a calculation of the diffraction pattern of the QC thas is modulated by a sine wave in superspace. With a superspace phonon, the energies involved at the intersection of the atomic surface with the cut and at the node of the atomic surface are totally different. One can thus not think of an atomic surface vibrating as whole with a given energy given by the superspace phonon, because such a picture does not differentiate the huge energy differences that come into play between different positions on the atomic surface. These energy differences also jeopardize the idea of a wavelength. In fact, in a real sine wave picture all points where the amplitude have the same value are conceptually energetically equivalent, while in the QC the corresponding local environments can be energetically totally different.

The term in the elastic tensor that is at stake is not one that tells you how one atom that moves along perpendicular space moves in response (along parallel space) to another atom that moves with a similar polarisation along perpendicular space. The real motion is along parallel space. In other words, if we want to use a picture of superspace elasticity, we must couple the atomic surfaces between each other uniquely along parallel space, and the coupling (or the potential energy) will vary along the atomic surface. With a sine wave representation there is no way how a derivative of the potential energy with respect to the perpendicular space displacement can be defined. The intermediate infinitesimal displacements needed to define such a derivative just do not exist: The displacement can only take two values: jump or no-jump. A use of this type of the concept of phason elasticity is therefore flawed. A jumping atom at the maximum in the double-well potential is not at all within the harmonic regime that is assumed when one defines elastic constants.

\section{B. Rigorous models}

\section{Model 1. The limit of small amplitudes}

Conceptual problems with very small amplitudes. Let us first develop a model wherein the amplitude of the distortion of the cut is bounded, i.e. $(\exists a)\left(\forall x_{\|}\right)\left(u_{\perp}\left(x_{\|}\right)<a\right)$. It is well known that an atomic surface can be subdived into disjoint parts, each one of which describes one precise local environment. For the Fibonacci chain, the central part of

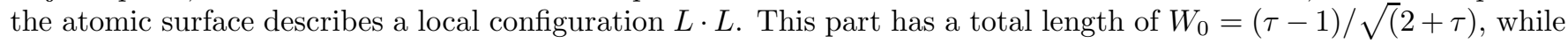
the total atomic surface has a length $W=(\tau+1) / \sqrt{(} 2+\tau)$. If we take a maximum amplitude of $\left(W-W_{0}\right) / 2$, the atoms that are in a first-neighbour environment $L \cdot L$ cannot jump. This subdivides the whole Fibonbacci chain in segments $L S L$ and $L S L S L$, between atoms that cannot jump, while within the segments jumps are possible. Within such a segment there can be correlations between the atomic jumps in the sense that e.g. in $L L S S L$, the atom between the two short distances will only be able to jump if another jump will have exchanged one of the two letters $S$ for an $L$. But there can be no correlations across the boundaries of the segments: In other words, the dynamics on the segments are mutually independent. The absence of such correlations over longer distances corresponds to the normal standard paradigm, that cannot be just ignored or questioned arbitrarily: Taking exception with it by postulating a possibility of additional correlations over long distances must be considered as a most unusual, awkward procedure. In a first approximation, we thus assume that we cannot have correlations across a local configuration $L \cdot L$.

It is already clear from this, that with the maximum amplitude choosen there can be no diffusion whatsoever, because all atomic motion remains confined within the segments. We will show also that the quasielastic signal that corresponds to such a model can be calculated and has a smooth $Q$-dependence, such that it cannot possibly correspond to the sine wave interpretation. Nonetheless, the sine wave interpretation has been postulated to be meaningful and straightforward by the authors, without any mention of a possible reserve about the generality of its validity, e.g. by warning that the amplitudes should not be taken too small.

As we will see, it is easy to map out the configuration spaces for the jump dynamics on the isolated segments, and due to the independence of the segments, it follows that the configuration space for the whole Fibonacci chain 
factorizes into a product of such segment configuration spaces.

Larger small amplitudes. One can relax the amplitude condition by going to the $\tau^{m}$ times inflated tiling, with $m$ even, and choose it such that only the atoms that are at a position $L L$ in the inflated tiling are not allowed to jump (When $m$ is odd, $L \cdot L$ of the inflated tiling corresponds to $L S \cdot L S$, such that the sectioning does not occur in an environment $L \cdot L)$. These atoms correspond to a $\tau^{m}$ times deflated domain on the atomic surface, such that a larger amplitude $\left(W-(\tau-1)^{m} W_{0}\right) / 2$ for the cut distortion can be accepted. The atomic motion will now be confined to $\tau^{m}$ times larger segments. This shows that we have to go to the limit of an amplitude that is equal to the whole length $W / 2$ before we can have unbounded, long-range diffusion. This raises the question: How large can the amplitudes become, before we start to see second-order satellites in the calculation of the sine wave modulation calculated above?

Calculation of the Dynamics for Isolated Segments LSLSL and LSL. As we already pointed out, the first step in the calculation of a coherent quasielastic signal is mapping out the configuration space. For the isolated segment $L S L$ the configuration space consists just of $S L L-L S L-L L S$, where the hyphens indicate that the configurations are "connected" in the sense that a jump with relaxation time $\tau_{0}$ permits to go (both ways) from one configuration to the other.

For the isolated segment $L S L S L$, the configuration space corresponds to all permutations of the letters in the five-letter word $L S L S L$ that lead to real changes. We can map it onto the problem of two "pseudoparticles" $S$ that diffuse on five pseudosites (the letters of the word $L S L S L$ ), where the letters $L$ would code an "empty pseudosite". These two pseudoparticles $S$ (1) cannot be simultaneously on the same site, and (2) they cannot leapfrog. If these two conditions were not necessary, the configuration space (with its connectivity) would be just a square lattice of $5 \times 5$ nodes $\left(j_{1}, j_{2}\right) \in(\mathbb{Z} \cap[1,5])^{2}$, where $j_{1}$ and $j_{2}$ label the respective positions of the two pseudoparticles. The jump matrix in configuration space would map then onto the jump matrix of the diffusion problem of an abstract particle (the system) that diffuses on this square lattice (the configuration space of our problem) between first-neighbour positions. The two conditions (1) and (2) correspond to exluding the line $j_{1}=j_{2}$, from this configuration space, and removing also one of the two triangular sections produced by this line, e.g. the triangular part $j_{1}>j_{2}$.

The problem of the pristine square lattice without fermionic constraint (which is thus is not sectioned by $j_{1}=j_{2}$ ) can be solved rigorously and algebraically by using translational invariance. Its 25 eigenvectors $\mathbf{v}^{\left(k_{1}, k_{2}\right)}$ are each defined by their 25 components $v_{j_{1}, j_{2}}^{\left(k_{1}, k_{2}\right)}=\cos \left(\frac{\pi}{10}\left(k_{1}-1\right)\left(2 j_{1}-1\right)\right) \cos \left(\frac{\pi}{10}\left(k_{2}-1\right)\left(2 j_{2}-1\right)\right)$. This means that each eigenvector has 25 components that we label by the compound indices $\left(j_{1}, j_{2}\right)$, where $j_{1}, j_{2}$ are integers within $[1,5]$. There are 25 eigenvectors that we label by the compound indxices $\left(k_{1}, k_{2}\right)$, where $k_{1}, k_{2}$ are integers within [1,5]. This is possible because the eigenvectors are the tensor products of the five five-dimensional eigenvectors $\mathbf{v}^{(k)}$ for the single-pseudoparticle diffusion problem, with components given by $v_{j}^{(k)}=\cos \left(\frac{\pi}{10}((k-1)(2 j-1))\right)$. The corresponding eigenvalues for the pristine square-lattice problem are $-\frac{4}{\tau_{0}}\left(\sin ^{2}\left(\frac{\pi}{20}\left(k_{1}-1\right)\right)+\sin ^{2}\left(\frac{\pi}{20}\left(k_{2}-1\right)\right)\right)$, i.e. sums of two eigenvalues of the single-pseudoparticle problem.

But when the fermionic condition has to be taken into account and the symmetry is broken by the cut $j_{1}=j_{2}$, the simple calculation is no longer possible. In fact, the eigenvalues have no longer any relation whatsoever with those of the problem on the square lattice.

Mathematical Formalism for Larger Isolated Segments. Going to higher levels of inflation $m$, with $m$ even, we will have $F_{n}$ letters $S$ and $F_{n+1}$ letters $L$ (for the larger segment), such that the problem can be mapped onto the diffusion problem of $F_{n}$ particles on $F_{n+2}$ sites. Here $n=m+3$ (e.g. for the $\tau^{2}$ inflated tiling, $m=2$ and we have $F_{5}=5$ particles on $F_{7}=13$ sites for the larger segment). The fact that the particles cannot be on a same site and cannot leapfrog implies that the number of configurations (on the larger segment) is $\left(\begin{array}{c}F_{n+2} \\ F_{n}\end{array}\right)$. On the smaller segment it is $\left(\begin{array}{c}F_{n+1} \\ F_{n-1}\end{array}\right)$. E.g. when $m=0$, we have $n=3$ for the segments $L S L S L$, with $\left(\begin{array}{c}F_{5} \\ F_{3}\end{array}\right)=\left(\begin{array}{c}5 \\ 2\end{array}\right)=10$ configurations, while for the segments $L S L$ we have $\left(\begin{array}{l}F_{4} \\ F_{2}\end{array}\right)=\left(\begin{array}{l}3 \\ 1\end{array}\right)=3$ configurations.

The $F_{n}$ particles will have coordinates $\left(j_{1}, j_{2} \cdots j_{F_{n}}\right) \in\left(\left[1, F_{n+2}\right] \cap \mathbb{Z}\right)^{F_{n}}$, which is a $F_{n}$-dimensional lattice bounded by a hypercube of edge length $F_{n+2}-1$. The condition that the particles cannot be on the same sites defines now forbidden hyperplanes $j_{1}=j_{2}, j_{2}=j_{3}, \cdots j_{F_{n}-1}=j_{F_{n}}$, which are sectioning the hypercubic lattice. Again, the diffusion problem on the hypercubic lattice could be easily solved in general by using translational invariance, but, again, the cuts by the hyperplanes break the symmetry and spoil any possibility of a general algebraic solution.

In any case, generally spoken, one will have to allow for segmentation lengths that are quite large before one will obtain very small non-zero eigenvalues. Finally, it may be noted it is not impossible that the condition 
$u_{\perp}\left(x_{\perp}\right) \leq\left(W-(\tau-1)^{m} W_{0}\right) / 2$, might remove more configurations from the hypercubic lattice than we described in terms of its sectioning with the hyperplanes $j_{1}=j_{2}, j_{2}=j_{3}, \cdots j_{F_{n}-1}=j_{F_{n}}$ above. But this is not important for the basic principle of the arguments we will develop below. The sizes of the configuration spaces we have considered above are then upper limits for the sizes we can expect. The numbers $n_{\nu}$ below have then to be replaced by smaller numbers.

Mathematical Formalism for a whole chain of isolated segments. The configuration space for the whole Fibonacci chain is a Cartesian product of the configuration spaces for segments of $F_{n}$ letters $S$ and $F_{n+1}$ letters $L$. The eigenvalues for the whole chain are the sums of the eigenvalues for each segment, and the eigenvectors are the tensor products of the eigenvectors of the segments. The fact that we cannot give the eigenvectors and eigenvalues for the problems on the isolated segments explicitly, does not impede one to develop the calculation further and to reach general conclusions for the dynamics of the whole Fibonacci chain based on such segments. The further calculation is analogous to the one in [22], which shows clearly that the coherent quasielastic signal has a smooth $Q$-dependence. Most importantly, the signal is not composed of more or less similar replicas around every Bragg, i.e. it does not show the salient hallmarks of a convolution product that are present in the data. In fact, the eigenvalues that are sums of eigenvalues of several single-segment problems remain silent.

We give here a shortened account of the calculation. The reader can compare it with the calculation in 22]. This should in principle permit him to understand the whole argument, even if at first sight it might look intimidating. Let us note a configuration on the Fibonacci chain as $\mathcal{C}_{\left\{j_{\nu}\right\}}=\mathcal{C} \cdots j_{1} j_{2} \cdots j_{\nu} \cdots$, which means that in segment $\nu \in \mathbb{Z}$ the configuration is $c_{j_{\nu}}$. As pointed out above, $j_{\nu}$ can take here any value from 1 to $n_{\nu}=\left(\begin{array}{c}F_{n+2} \\ F_{n}\end{array}\right)$, for the larger segment, and from 1 to $n_{\nu}=\left(\begin{array}{c}F_{n+1} \\ F_{n-1}\end{array}\right)$, for the smaller segment. Here $n-3=m$ is the (necessarily even) inflation level.

The Fourier transform of the configuration on the Fibonacci chain is:

$$
\mathcal{F}\left(\mathcal{C}_{\left\{j_{\nu}\right\}}\right)=\sum_{\nu \in \mathbb{Z}} \tilde{\mathcal{F}}\left(c_{j_{\nu}}\right) e^{\imath Q x_{\nu}}
$$

where $x_{\nu}$ is the left boundary of the segment $\nu$, and $\tilde{\mathcal{F}}\left(c_{j_{\nu}}\right)$ is the Fourier transform of the configuration on the segment calculated in relative coordinates $x-x_{\nu}$. The eigenvectors $\mathbf{v}^{\left\{k_{\nu}\right\}}=\mathbf{v}^{\left(\cdots k_{1} k_{2} \cdots k_{\nu} \cdots\right)}$ are tensor products

$$
\mathbf{v}^{\left\{k_{\nu}\right\}}=\bigotimes_{\nu \in \mathbb{Z}} \mathbf{v}^{\left(k_{\nu}\right)}
$$

such that their components are of the form $v_{\left\{j_{\nu}\right\}}^{\left\{k_{\nu}\right\}}=v_{\cdots j_{1} j_{2} \cdots j_{\nu} \cdots}^{\left(\cdots k_{1} k_{2} \cdots k_{\nu} \cdots\right)}=\cdots v_{j_{1}}^{\left(k_{1}\right)} v_{j_{2}}^{\left(k_{2}\right)} \cdots v_{j_{\nu}}^{\left(k_{\nu}\right)} \ldots$. Note that $k_{\nu}$ are here just labels for the eigenvectors, and that the eigenvectors are not at all assumed to be of the translationally invariant type $v_{j_{\nu}}^{\left(k_{\nu}\right)}=\cos \left(\frac{\pi}{2 N_{\nu}}\left(\left(k_{\nu}-1\right)\left(2 j_{\nu}-1\right)\right)\right)$, where $N_{\nu}$ would take the value $F_{n+1}$ or $F_{n}$ depending on the type of segment. However it is assumed that by definition the label $k_{\nu}=1$ corresponds to the eigenvalue 0 and that the corresponding eigenvector is then $[1,1, \cdots 1]^{\top}$, which will e.g. be the case if all jump times in the dynamics on a single segment are the same. (Actually it is sufficient that all jumps take place in symmetrical double-well potentials). We must calculate

$$
\sum_{\left\{j_{\nu}\right\}} \mathcal{F}\left(\mathcal{C}_{\left\{j_{\nu}\right\}}\right) v_{\left\{j_{\nu}\right\}}^{\left\{k_{\nu}\right\}}=\sum_{\left\{j_{\nu}\right\}}\left(\sum_{w \in \mathbb{Z}} \tilde{\mathcal{F}}\left(c_{j_{w}}\right) e^{\imath Q x_{w}}\right)\left(\prod_{\nu} v_{j_{\nu}}^{\left(k_{\nu}\right)}\right) .
$$

This is a sum over $w$. The term $w$ is given by

$$
\prod_{\nu \neq w}\left(\sum_{j_{\nu}} v_{j_{\nu}}^{\left(k_{\nu}\right)}\right)\left(\sum_{j_{w}} \tilde{\mathcal{F}}\left(c_{j_{w}}\right) e^{\imath Q x_{w}} v_{j_{w}}^{\left(k_{w}\right)}\right)=\left(\prod_{\nu \neq w} \sqrt{n_{\nu}} \delta_{k_{\nu} 1}\right)\left(\sum_{j_{w}} \tilde{\mathcal{F}}\left(c_{j_{w}}\right) e^{\imath Q x_{w}} v_{j_{w}}^{\left(k_{w}\right)}\right) .
$$

When $k_{\nu}=1$ on segment $\nu$, the eigenvalue is 0 and the normalized eigenvector has $n_{l}$ identical components $1 / \sqrt{n_{l}}$ on the segment, which explains the prefactor $\sqrt{n_{\nu}}$ of $\delta_{k_{\nu}}$. We do not have to care too much about these prefactors, since after squaring amplitudes, and weigthing all configurations to the same initial probability, they will disappear from the final result.

The sum over $w$ is only non-zero in two cases. The first case is that $(\forall \nu \in \mathbb{Z})\left(k_{\nu}=1\right)$, which leads to the eigenvalue $\lambda=0$, with the corresponding eigenvector whose components are all equal to $1 / \prod_{\nu} \sqrt{n_{\nu}}$. Leaving apart this normalization factor, as it will eventually disappear anyway, we obtain the result: 


$$
\sum_{w} \sum_{j_{w}} \tilde{\mathcal{F}}\left(c_{j_{w}}\right) e^{\imath Q x_{w}}
$$

which is just the sum of the Fourier transforms of all random tiling configurations. The second case is that $(\exists$ ! $w \in$ $\mathbb{Z})\left(k_{w} \neq 1\right) \&(\forall \nu \neq w)\left(k_{\nu}=1\right)$. Then the eigenvalue will be $\lambda_{w}$ and the result will reduce to just one term:

$$
\sum_{j_{w}} \tilde{\mathcal{F}}\left(c_{j_{w}}\right) e^{\imath Q x_{w}} v_{j_{w}}^{\left(k_{w}\right)}
$$

Apart from the phase factor, $e^{\imath Q x_{w}}$, this is exactly the expression for the analogous dynamical problem for just the isolated segment $w$. Carrying the calculation through until the end we must take the squares of the amplitudes. We get then an elastic term (a constant in the time domain, or $\delta(\omega)$ in the energy domain), that is weighted with the (averaged) diffraction intensity of the random tiling model, and $\left(\begin{array}{c}F_{n+2} \\ F_{n}\end{array}\right)+\left(\begin{array}{c}F_{n+1} \\ F_{n-1}\end{array}\right)-2$ quasielastic terms $e^{-\lambda_{w} t}$ (in the time domain) that are weighted with structure factors that have exactly the same $Q$-dependence as in the dynamical jump models for the small and the large single segments. We can understand the result also in an intuitive way, arguing that the segment dynamics are independent.

Postulating correlations by brute force? When $a<W / 2$ one can of course argue, that the atomic positions in the various segments can be correlated anyway in a sine wave fashion, but (1) this contradicts the accepted paradigm of the stochastic nature of atomic jumps in alloys, (2) reverses the charge of proof and (3) cannot cover up the fact that the authors completely missed this point. The exceptional character of such a claim should at least have been pointed out explicitly instead as presenting it as a trivial and self-evident.

We may note that with large isolated fragments, we allow already for large distances of correlation, and that we have shown for these cases that the signal lacks the hallmarks of a convolution product in the form of replicas around each Bragg peak. One could argue also that the correlations aimed at are of a different nature than the ones we have explored. But this can only show how it even has not been defined what the as self-evident presented correlations are supposed to be. In our opinion postulating correlations of another type than we have allowed for (1) must be clearly defined, (2) properly justified to be physically plausible, and (3) it must be proved by a model calculation that it leads indeed to the type of signal observed.

\section{Model 2. The insolvable limit of large amplitudes and configuration space percolation}

When $a>W / 2$, the configuration space is no longer factorized. We are then entitled to hope that small eigenvalues will enter the game. But we may note that the relative proportions of letters $S$ and $L$ render it very doubtful that the eigenvectors and eigenvalues could be reasonably approximated by the solution that one could propose for a small number $m$ of particles on an infinite lattice, viz. by considering that it is to a high degree of accuracy the tensor product of $m$ eigenvectors for the single-particle problem. For these reasons the Ansatz of the authors that the eigenvalues would be of the type that leads within the energy domain to a Lorentian signal with a width $D Q^{2}$ lacks a proper justification. In any case, this model case is of an unassailable difficulty. The same can be said about models for two- and three-dimensional quasicrystals.

One can consider the Fibonacci chain as the limit of a finite diffusion problem, whose size tends to infinity. In the finite problem there will be $\beta$ letters $S$, and $\xi-\beta$ letters $L$. The phason jumps dynamics can then be formulated as the jump diffusion problem of $\beta$ particles (the letters $S$ ) on $\xi$ sites (the total number of letters), as we already did above for models 1 and 2. (Alternatively, it can be formulated as the jump diffusion problem of $\xi-\beta$ particles (the letters $L$ ) on $\xi$ sites. The solutions will be the same). All one-dimensional diffusion problems with $\beta$ diffusing particles on $\xi$ sites (where $\xi \geq \beta$ ) can be handled by using the irreducible representations of the permutation group $S_{\xi}$ : Each of the $\xi-1$ generators $\left(\begin{array}{cc}j & j+1 \\ j+1 & j\end{array}\right)$, (with $j \in[1, \xi-1] \cap \mathbb{N}$ ), of the symmetric group $S_{\xi}$ interchanges the occupation of two adjacent sites $j$ and $j+1$. Some of these interchanges lead to real meaningful physical changes, other ones do not, because they lead to physically identical configurations. All identical configurations build an equivalence class. The question which permutations lead to identical configurations decides in a further stage which ones of the $\xi$ ! eigenvectors that one can derive from the permutation group, remain silent in the final signal. In fact, it can be shown that in order to obtain the solution of the physical problem it suffices to solve the problem over the permutation group, (i.e. by considering all permutations to be different, despite the fact that some of them correspond to configurations that are physically equivalent), and to sum afterwards over all configurations 
that are physically equivalent. (The corresponding summing over the eigenvectors will in certain cases lead to zero vectors, such that the result is no longer an eigenvector, and the original eigenvalue ceases to be an eigenvalue). With $\beta$ particles on $\xi$ sites we will have to sum over the $\beta$ ! permutations of identical particles and over the $(\xi-\beta)$ ! permutations of empty sites. In other words, each equivalence class has cardinal $\beta$ ! $(\xi-\beta)$ !. (The equivalence of the solutions for $\beta$ or $\xi-\beta$ diffusing particles mentioned above is due to the fact that they result in identical sums). One of the steps in this proof consists in showing that by summing the differential equations over these $\beta$ ! $(\xi-\beta)$ ! permutations we obtain the correct differential equations for the equivalence classes. The irreducible representations of the symmetric group $S_{\xi}$ are calculated on the basis of Young tableaus. For small values of $\xi$, this embedding of the problem within the permutation symmetry group allows one to tackle the jump diffusion problems on a case by case and representation by representation basis. (Even in the simple case of the diffusion of a single particle, the solutions are scattered over the various irreducible representations). But the possible dimensions of the irreducible representation matrices grow with $\xi$, and it is not obvious to see relationships that would permit us to link the calculations for $\xi$ with those for $\xi+1$. In other words, it is hard to see how one could derive from this formalism a scheme that would permit us to find nice, general algebraic expressions for the eigenvalues and eigenvectors in terms of $\beta$ and $\xi$ in a way that would be analogous to the expressions we were able to derive above (in the discussion of model 1) in the presence of translational invariance, with its uniquely one-dimensional irreducible representations. This hypothetical scheme would then have to serve as a platform for taking the limit of the results when $\beta \rightarrow \infty$ and $\xi \rightarrow \infty$, keeping the ratio $\beta / \xi$ constant. The fact that the symmetry group that underlies the jump models is the permutation group, and that the irreducible representations of the permutation groups can reach arbitrarily high dimensions thwarts the aspirations of finding simple, universal expressions for solutions of model 2.

\section{Model 3. Diffusing tiles}

Introduction. Let us assume that on the Fibonacci chain a letter $S_{1}$ is allowed to diffuse, by processes $L S \leftrightarrow S L$ until it becomes the neighbour another letter $S_{2}$, in a situation $L S_{1} S_{2} L$. Then only two possibilities are possible, viz. backwards to $S_{1} L S_{2} L$ or forwards to $L S_{1} L S_{2}$. In the latter case we assume that $S_{2}$ takes over the active rôle of $S_{1}$. Locally, the configuration space takes the topology of a linear chain: $\Leftrightarrow \cdots S L . L S L S L . L S \cdots \Leftrightarrow \cdots L S . L S L S L . L S \cdots \Leftrightarrow$ $\cdots L . L S S L S L . L S \cdots \Leftrightarrow \cdots L . L S L S S L . L S \cdots \Leftrightarrow \cdots L . L S L S L S . L S \cdots \Leftrightarrow \cdots L . L S L S L . L S S \cdots \Leftrightarrow$. Moreover we see that when we go from the left to the right in this sequence, the Fibonacci chain is step by step translated to the left, as the segments are recovered (with a shift of one letter to the left), after the passage of the active letter(s) $S$. We see that this is a many-particle jump model. The model is diffusive. The single particles do not travel over long distances. It is rather a pseudo-particle, the active letter $S$, that undergoes long-range diffusion. From the viewpoint of this pseudo-particle, the model has all the characteristics of a single-particle model. If we allow only one tile or one letter $S$ to diffuse this way, the model is perhaps not very realistic, but (1) it is the only case that is simple enough to permit a rigorous calculation and (2) it leads to remarkable results for the coherent scattering signals, from which important lessons can be learned. We can split this model into two submodels according to the philosophy of approach.

Philosophy 1. In this philosophy we may assume that the error that initiated the motion of the active letter $S$ is at $-\infty$, such that there is no trace of the beginning of the process. The se-


$\cdots L . L S L S L S . L S \cdots \Leftrightarrow \cdots L . L S L S L . L S S \cdots \Leftrightarrow$ mentioned above is then unbounded, both to the left and to the right, and the topology of configuration space is identical to that of a linear chain. The configurations $\mathcal{C}_{j}$ are of the type $c_{1} S c_{2}$, where $c_{1} c_{2}$, is the full Fibonacci sequence, and $S$ is the active letter $S$. The jump model is defined by $(\forall j \in \mathbb{Z})\left(\frac{\partial p_{j}}{\partial t}=\frac{1}{\tau_{0}}\left(p_{j-1}-2 p_{j}+p_{j+1}\right)\right.$ (where $p_{j}$ denotes the probability that the QC is in the configuration $\left.\mathcal{C}_{j}\right)$. The eigenvectors and eigenvalues of the "infinite jump matrix" are therefore straightforward to calculate, and the Fourier transforms of the configurations also. The eigenvectors $\mathbf{v}^{(q)}$ have components $(\forall j \in \mathbb{Z})\left(v_{j}=e^{\imath q(j-1)}\right)$, where $q \in \mathbb{R}$. The corresponding eigenvalues are $\lambda_{q}=-\frac{4}{\tau_{0}} \sin ^{2} \frac{q}{2}$. But as the assumption that there is no trace of the beginning of the process is artificial, we will rather calculate the result for the following model, that contains all the difficulties on a larger scale.

Philosophy 2. In this philosophy we assume that we start from a perfect Fibonacci chain, and initiate the dynamics with a first jump of a letter $S$, at some point $P$ after which we allow this letter $S$ to diffuse further. This initial jump leaves a scar behind in the Fibonacci chain that permits to spot the point $P$ where the whole process started, e.g. by creating a local sequence $L L L$ that will not occur anywhere else in the chain. When the active letter accidently gets back to the point $P$, this scar may be removed, undoing all traces of the previous history. We recover the perfect Fibonacci chain and allow the process to restart from a completely different place. The configurations $\mathcal{C}$ are of the type $c_{1} c_{2} S c_{3}$, where $c_{1} S c_{2} c_{3}$, is the full Fibonacci sequence, and $S$ is the active letter $S$. In this case the topology of configuration space is starlike. A central point $O$ that corresponds to the perfect Fibonacci lattice is the origin of an infinity of half linear chains. Each half linear chain is defined by an aggregate of two parameters, viz. the starting point and the direction (left or right) of diffusion of the active letter $S$. We label the direction by $\epsilon \in\{-1,1\}$, and 
the starting point by $m \in \mathbb{Z}$. Let us call the perfect lattice the configuration $\mathcal{C}_{0}$. All other configurations can then be labeled as $\mathcal{C}_{m, \epsilon, j}$, where $(m, \epsilon)$ labels the half linear chain, and $j$ the position on the half linear chain starting from $\mathcal{C}_{0}$. The Fourier transforms of all the intervening configurations on the Fibonacci chain are still easy to calculate. But the starlike topology of configuration space implies that the infinite jump matrix can no longer be diagonalized by using translational invariance. In fact, the eigenvalue equations for the probabilities $p_{m, \epsilon, j}$ that the system is in the configuration $\mathcal{C}_{m, \epsilon, j}$ are:

$$
\begin{gathered}
(\forall j \in \mathbb{N} \| j>1)(\forall m \in \mathbb{Z})(\forall \epsilon \in\{-1,1\})\left(\lambda p_{m, \epsilon, j}=\frac{1}{\tau_{0}}\left(p_{m, \epsilon, j-1}-2 p_{m, \epsilon, j}+p_{m, \epsilon, j+1}\right)\right), \\
(\forall m \in \mathbb{Z})(\forall \epsilon \in\{-1,1\})\left(\lambda p_{m, \epsilon, 1}=\frac{1}{\tau_{0}}\left(p_{0}-2 p_{m, \epsilon, 1}+p_{m, \epsilon, 2}\right)\right), \\
\lambda p_{0}=\sum_{m \in \mathbb{Z}} \sum_{\epsilon \in\{-1,1\}}\left(p_{m, \epsilon, 1}-p_{0}\right) .
\end{gathered}
$$

Eigenvalues and eigenvectors of the model. The diagonalization can be done rigorously by using the transfer matrix method on each of the half linear chains. In fact, the first equation that defines the eigenvalue problem of the jump matrix can be solved for $p_{m, \epsilon, j+1}$ in terms of $p_{m, \epsilon, j}$ and $p_{m, \epsilon, j-1}$, such that in the end, using this as a recurrence relation, all $p_{m, \epsilon, j+1}$ can be expressed in terms of $p_{m, \epsilon, 1}$ and $p_{0}$. The recurrence equation is $p_{m, \epsilon, j+1}=\left(\lambda \tau_{0}+2\right) p_{m, \epsilon, j}-p_{m, \epsilon, j-1}$. The required expression is derived by solving the characteristic equation $\mu^{2}=\left(\lambda \tau_{0}+2\right) \mu-1$. This equation has a double root $\left(\mu_{1}=\mu_{2}=1\right)$ when $\lambda \tau_{0}=0$. The solution is then of the form $p_{m, \epsilon, j}=K_{m, \epsilon, 1}+j K_{m, \epsilon, 2}$. This remains only finite when $j \rightarrow \infty$ when $K_{m, \epsilon, 2}=0$, such that $(\forall j \in \mathbb{N})\left(p_{m, \epsilon, j}=K_{m, \epsilon, 1}\right)$, from which it follows that also $p_{0}=K_{m, \epsilon, 1}$. Hence the unique eigenvector that corresponds to $\lambda=0$ takes the same constant value in all its components. When the equation has two distinct roots, the solution is of the form $p_{m, \epsilon, j}=K_{m, \epsilon, 1} \mu_{1}^{j}+K_{m, \epsilon, 2} \mu_{2}^{j}$, where $K_{m, \epsilon, 1}$ and $K_{m, \epsilon, 2}$ are determined by $p_{0}$ and $p_{m, \epsilon, 1}$. This leads to

$$
p_{m, \epsilon, j}=p_{m, \epsilon, 1} \frac{\mu_{1}^{j}-\mu_{2}^{j}}{\mu_{1}-\mu_{2}}-p_{0} \frac{\mu_{1}^{j-1}-\mu_{2}^{j-1}}{\mu_{1}-\mu_{2}} .
$$

As $\mu_{1} \mu_{2}=1$, the solution will only remain finite when $j \rightarrow \infty$ if $\left|\mu_{1}\right|=\left|\mu_{2}\right|=1$. By putting $\mu_{1}=e^{\imath q}$, with $\left.q \in\right] 0, \pi[$ we obtain $\lambda \tau_{0}=-4 \sin ^{2} \frac{q}{2}$, and thus:

$$
p_{m, \epsilon, j}=p_{m, \epsilon, 1} \frac{\sin j q}{\sin q}-p_{0} \frac{\sin (j-1) q}{\sin q} .
$$

The wole difficulty resumes then to finding the set of values $p_{0}, p_{m, \epsilon, 1}$, satisfying Eq. (11), that defines a complete orthogonal set of eigenvectors for each value of $\lambda \neq 0$. Note that we must take a maximal number of mutually independent solutions of Eq. (11), and that these solutions must simultaneously lead to linear independent eigenvectors. We will first act as though the number of quantities $p_{m, \epsilon, 1}$ were a finite number $2 N$, and then generalize towards an infinite vector space. In other words, the star with an infinite number of half chains is taken as the limit of an $2 N$-branched star, when $N \rightarrow \infty$. We will use a new type of labeling. For $\epsilon=1$ we will label $(m, \epsilon)$ by $m$, and for $\epsilon=-1$ we will note $(m, \epsilon)$ by $N+m$. For convenience we will use an intermediary basis for the vectors $\mathbf{p}=\left(p_{0}, p_{1,1}, p_{2,1}, \cdots, p_{m, 1}, \cdots, p_{2 N, 1}\right)$. Let us note the canonical basis as $\mathbf{g}_{0}, \mathbf{g}_{1}, \cdots, \mathbf{g}_{m}, \mathbf{g}_{2 N}$.

We define this intermediary basis as follows: 


$$
\begin{array}{ll}
\mathbf{e}_{0} & =(1,0,0,0, \cdots, 0) \\
\mathbf{e}_{1} & =\left(\mathbf{g}_{1}-\mathbf{g}_{N+1}\right) / \sqrt{2} \\
\mathbf{e}_{2} & =\left(\mathbf{g}_{2}-\mathbf{g}_{N+2}\right) / \sqrt{2} \\
\vdots & \\
\mathbf{e}_{m} & =\left(\mathbf{g}_{m}-\mathbf{g}_{N+m}\right) / \sqrt{2} \\
\vdots & \\
\mathbf{e}_{N} \quad=\left(\mathbf{g}_{N}-\mathbf{g}_{2 N}\right) / \sqrt{2} \\
\mathbf{e}_{N+1}=(0,1,1,1, \cdots, 1) / \sqrt{2 N} \\
\mathbf{e}_{N+2}=\left(0,1, e^{\imath \psi}, e^{\imath 2 \psi}, \cdots, e^{\imath(r-1) \psi}, \cdots, e^{\imath(N-1) \psi}, 1, e^{\imath \psi}, e^{\imath 2 \psi}, \cdots, e^{\imath(r-1) \psi}, \cdots, e^{\imath(N-1) \psi}\right) / \sqrt{2 N} \\
\quad=\sum_{r=1}^{N} e^{\imath(r-1) \psi}\left(\mathbf{g}_{r}+\mathbf{g}_{N+r}\right) / \sqrt{2 N} \\
\mathbf{e}_{N+m}=\sum_{r=1}^{N} e^{\imath(r-1)(m-1) \psi}\left(\mathbf{g}_{r}+\mathbf{g}_{N+r}\right) / \sqrt{2 N} \\
\vdots \\
\mathbf{e}_{2 N}=\sum_{r=1}^{N} e^{\imath(r-1)(N-1) \psi}\left(\mathbf{g}_{r}+\mathbf{g}_{N+r}\right) / \sqrt{2 N}
\end{array}
$$

This is an orthonormal basis (We must take $\psi=2 \pi / N$ in the finite case). Let us call the components of $\mathbf{p}$ in this basis $\gamma_{m}$, such that $\mathbf{p}=\sum_{m} \gamma_{m} \mathbf{e}_{m}$. A first eigenvector of our problem corresponds to $\lambda=0$, and has the form $(1,1,1, \cdots 1,1)=\mathbf{e}_{0}+\sqrt{2 N} \mathbf{e}_{N+1}$.

Assume first that $p_{0}=0$. Then $\gamma_{0}=\mathbf{p} \cdot \mathbf{e}_{0}=p_{0}=0$. Eq. (11) becomes then $0=\sum_{m \neq 0} \sum_{\epsilon \in\{-1,1\}} p_{m, \epsilon, 1}=$ $\mathbf{p} \cdot \mathbf{e}_{N+1} \sqrt{2 N}=\gamma_{N+1}$. The fact that $\gamma_{0}=\gamma_{N+1}=0$ implies that the vectors $\mathbf{p}$ that satisfy Eq. (11) all belong to the subspace spanned by the vectors $\mathbf{e}_{1}, \mathbf{e}_{2}, \ldots \mathbf{e}_{N}, \mathbf{e}_{N+2}, \mathbf{e}_{N+3}, \ldots \mathbf{e}_{2 N}$. In fact, each of these basis vectors alone satisfies the condition Eq. (111). Note that the vectors $\mathbf{p}$ are not eigenvectors of our problem, but serve to define the eigenvectors, by fixing the values of $p_{0}$ and $p_{m, \epsilon, 1}$ that are needed to calculate all other components $p_{m, \epsilon, j}$ of an eigenvector by using Eq. (13).

For a given non-zero eigenvalue $\lambda$ we have this way already found $2 N-2$ linearly independent, and mutually orthogonal eigenvectors with the choice $p_{0}=0$. They are of the form $\mathbf{e}_{m} \otimes(\sin q, \sin 2 q, \cdots, \sin j q, \ldots)$. Let us now check what additional eigenvectors we can find by supposing that $p_{0} \neq 0$. Eq. (11) becomes then $\left(\sum_{m} \sum_{\epsilon \in\{-1,1\}}\left(p_{m, \epsilon, 1}-p_{0}\right)\right)-\lambda p_{0}=0$. Now $p_{m, \epsilon, 1}-p_{0}$ are the components of the vector $\mathbf{p}-p_{0} \mathbf{e}_{0}-\sqrt{2 N} p_{0} \mathbf{e}_{N+1}$. Let us note this quantity as $\sum_{m} \gamma_{m} \mathbf{e}_{m}$. Then $\lambda p_{0}=\sum_{m, \epsilon}\left(p_{m, \epsilon, 1}-p_{0}\right)=(1,1,1 \cdots) \cdot\left(\mathbf{p}-p_{0} \mathbf{e}_{0}-\sqrt{2 N} p_{0} \mathbf{e}_{N+1}\right)=\left(\mathbf{e}_{0}+\right.$ $\left.\sqrt{2 N} \mathbf{e}_{N+1}\right) \cdot\left(\mathbf{p}-p_{0} \mathbf{e}_{0}-\sqrt{2 N} p_{0} \mathbf{e}_{N+1}\right)=\gamma_{0}+\sqrt{2 N} \gamma_{N+1}$. Now $\mathbf{e}_{0} \cdot \mathbf{p}=p_{0}$, such that $\gamma_{0}=\mathbf{e}_{0} \cdot\left(\mathbf{p}-p_{0} \mathbf{e}_{0}-\sqrt{2 N} p_{0} \mathbf{e}_{N+1}\right)=$ 0 . Hence $\gamma_{N+1}=\lambda p_{0} / \sqrt{2 N}$, and $\mathbf{p}-p_{0} \mathbf{e}_{0}-\sqrt{2 N} p_{0} \mathbf{e}_{N+1}=\left(0, \gamma_{1}, \gamma_{2}, \gamma_{3}, \cdots \gamma_{N}, \lambda p_{0} / \sqrt{2 N}, \gamma_{N+2}, \gamma_{N+3}, \cdots \gamma_{2 N}\right)$, where the $\gamma_{m}$ are completely free. In the intermediary basis $\mathbf{p}$ has thus coordinates $\left(p_{0}, \gamma_{1}, \gamma_{2}, \cdots \gamma_{N}, p_{0} \sqrt{2 N}[1+\right.$ $\left.\lambda /(2 N)], \gamma_{N+2}, \gamma_{N+3}, \cdots \gamma_{2 N}\right)$.

If we construct additional eigenvectors with $\left(p_{0}, \gamma_{1}, \gamma_{2}, \cdots \gamma_{N}, p_{0} \sqrt{2 N}[1+\lambda /(2 N)], \gamma_{N+2}, \gamma_{N+3}, \cdots \gamma_{2 N}\right)$, then they should be orthogonal to the $2 N-2$ ones we already derived. The Gramm-Schmidt procedure will therefore reduce these additional eigenvectors to the ones that one can construct from $\left(p_{0}, 0,0, \cdots, 0,(1+\lambda /(2 N)) \sqrt{2 N} p_{0}, 0,0, \cdots, 0\right)$. As the eigenvectors are defined up to an arbitrary normalization constant, we can take e.g. $p_{0}=1$. Developing this in the pristine canonical basis, the component from $\mathbf{e}_{N+1}$ yields $1+\lambda /(2 N)$ on all $p_{m, \epsilon, 1}$. We can verify that with this choice of $\mathbf{p}$ for a given value $\lambda$, Eq. (11) is indeed satisfied and yields the eigenvalue $\lambda$. When $N \rightarrow \infty$ however, all $p_{m, \epsilon, 1} \rightarrow 1$, which corresponds to the eigenvector that goes with the eigenvalue $\lambda=0$. Hence, in the limit $N \rightarrow \infty$, only the eigenvalue $\lambda=0$ can have an eigenvector with the structure that corresponds to $p_{0} \neq 0$. Hence the choices $\mathbf{e}_{1}, \mathbf{e}_{2}, \ldots$ for $\mathbf{p}$, based on $p_{0}=0$, lead to a maximal set of mutually independent solutions for the constraint given by Eq. (11). By the choice of the intermediary basis, whole lines (rather than half lines) are always treated at once, and the solutions are eighter symmetrical $(s=1)$ or antisymmetrical $(s=-1)$ in $j$. This leads to eigenvectors $\mathbf{p}^{(k, s, q)}$, with $k \in \mathbb{Z}, q \in] 0, \pi[, s \in\{-1,1\}$ given by:

$$
\mathbf{p}^{(k, s, q)}=\left(1, \frac{\sin 2 q}{\sin q}, \cdots \frac{\sin j q}{\sin q}, \cdots\right) \otimes \mathbf{e}_{k, s},
$$

where the antisymmetrical eigenvectors $\mathbf{e}_{k, 1}$ are the generalisation of $\mathbf{e}_{k}$, with $0<k \leq N$ in the finite case, while the symmetrical eigenvectors $\mathbf{e}_{k,-1}$ are the generalisation of $\mathbf{e}_{k}$, with $N+1<k \leq 2 N$. If we take $j \in \mathbb{Z}$, we can write the components as:

$$
\left(p^{(k,-, q)}\right)_{m, j}=\frac{\sin j q}{\sin q} \delta_{k, m},
$$




$$
\left(p^{(k,+, q)}\right)_{m, j}=\frac{\sin |j| q}{\sin q} e^{\imath m \psi_{k}},
$$

which are mutually orthogonal by construction (and where the factor $1 / \sin q$ can be dropped if $q \neq 0$. For $q=0$, we have $\lambda=0$ and the eigenvector is $(1,1,1, \cdots, 1,1, \cdots)$. We can take $\psi_{k} \notin \pi \mathbb{Q}$. The use of $k$ is somewhat artificial and serves only to label the eigenvectors, which are defined by $\left.\psi_{k} \in\right]-\pi, \pi[$.

Calculation of the coherent scattering signal. We now must apply the formalism of Reference 20] to calculate the structure factors that go with each eigenvalue $\lambda$ and the time dependence $e^{-\lambda t}$. Let us note that in the limit $q \rightarrow 0, \lambda=-q^{2} / \tau_{0}$ to a first approximation, such that the width of the Lorentzian obtained from the Fourier transform of $e^{-\lambda t}$, will be of the type $D q^{2}$. For a given eigenvector $\mathbf{v}_{\lambda}$ that corresponds to an eigenvalue $\lambda$, there will be a contribution $e^{-\lambda t}$ to the signal with a weight given by $\left|\sum_{\mathcal{C}} \mathcal{F}(\mathcal{C}) \mathbf{v}_{\lambda}(\mathcal{C})\right|^{2}$, where the components $\mathbf{v}_{\lambda}(\mathcal{C})$ of the eigenvector taken at the configuration $\mathcal{C}$, run through the whole starlike graph of the configuration space. (It is thereby assumed, without loss of generality that all initial configurations have the same probability. In fact, we should not forget that the infinite configuration space is only the abstraction of a mathematical limit that is supposed to correspond to the huge numbers of atoms that are involved in a macroscopic solid. A real QC is always finite, and its configuration space also. That the initial configurations all have the same probability will be true for all real cases, i.e. for all stars with a finite number of branches whose length is also finite. By taking the limit when the number and the length of the branches tend to infinity, we will obtain a constant probability for the initial configurations all over the infinite star. Of course, one could also try to assess the true energies involved in the configurations in order to calculate their thermodynamical probabilities. But this corresponds to a level of precision and complexity that is far beyond the scope and the aims of our monoatomic toy model). We must take into account the occurrence of the modulus in $\sin |j| q$, and the fact that sometimes $\psi$ is not a parameter, but in all calculations the sum will contain two building bricks of the type:

$$
F^{(k, q)}=\sum_{m \in \mathbb{Z}} \sum_{j \in \mathbb{N}} \mathcal{F}\left(\mathcal{C}_{m, j}\right) e^{\imath m \psi} \sin j q .
$$

(The case when $\psi$ is not a parameter can be treated with the same formalism by putting $\psi=0$ ).

For this we must calculate the Fourier transforms $\mathcal{F}\left(\mathcal{C}_{m, j}\right)$ of all configurations $\mathcal{C}_{m, j}$. A configuration is defined by the starting point where the first flip away from the perfect tiling occurred and the endpoint, i.e. the actual position of the active, diffusing letter $S$. We can describe these two points by decomposing the letter sequence of the perfect lattice $\mathcal{C}_{0}$ as $c_{1} S c_{2} c_{3}$, where $S$ denotes the tile that is going to wander, and where the configuration reached $\mathcal{C}_{m, j}$ will be $c_{1} c_{2} S c_{3}$. We note the endpoint of $c_{1}$ as $P$ and the point between $c_{2}$ and $c_{3}$ by $B$. We call the point between $S$ and $c_{2}$ in the perfect tiling $A$. (We have $x_{B}>x_{A}$ ). The global change from $\mathcal{C}_{0}$ to $\mathcal{C}_{m, j}$ consists in: (1) removing the atom at $P,(2)$ moving all the atoms of $c_{2}$ over a distance of $\sigma=\frac{1}{\sqrt{2+\tau}}$ to the left, such that $A$ ends up in $P,(3)$ putting the atom we removed in $P$ at the position $B$ where the atom that initially occupied it has moved away over $\sigma$ to the left. (Here $\sigma$ stands for the length of the interval spanned by a letter $S$ ). All other atoms in $c_{1}$ and $c_{3}$ remain unchanged. The Fourier transform $\mathcal{F}\left(\mathcal{C}_{m, j}\right)$ of $\mathcal{C}_{m, j}$ can thus be written as:

$$
\mathcal{F}(\mathbb{Q} \mathbb{C})+\mathcal{F}\left(\mathbb{Q} \mathbb{C} \cdot\left(e^{-\imath Q \sigma}-1\right) \cdot \chi[A B]\right)+e^{\imath Q x_{B}}-e^{\imath Q x_{A}},
$$

where $\chi[A B]$ is the characteristic function of the interval $[A B]$, i.e. the function that takes the value 1 on the interval and the value 0 elsewhere. When the tile diffuses to the left $e^{-\imath Q \sigma}$ must be replaced by $e^{\imath Q \sigma}$, while the definitions of $x_{B}$ and $x_{A}$ must be maintained such that now $x_{B}<x_{A}$. The points $A$ correspond to the index $m$, the points $B$ correspond to the index $j$. We must now establish the relation that defines this correspondence. There is a complication when we try to number the configurations, in that it is not just a numbering of the letters of the Fibonacci sequence. In fact, when we have $S^{*} L S L S \rightarrow L S^{*} S L S\left(=L S S^{*} L S\right) \rightarrow L S L S^{*} S\left(=L S L S S^{*}\right)$, the active letter $S^{*}$ has jumped from the first to the fifth position in only two moves. It is therefore better to count the configurations by using the $\tau$-inflated quasicrystal. Let us note the letters of the non-inflated QC by $\ell$ ans $s$, and those of the inflated QC by $L$ and $S$. Then $L$ corresponds to $\ell s$ and $S$ corresponds to $\ell$. The dynamical sequence evoked can then be rewritten as $s L L \rightarrow L s L \rightarrow L L s$, where we reserve the use of the letter $s$ for the active letter $S^{*}$. Hence the index $j$ can be established by counting the letters in the inflated tiling. When a lattice point of the inflated tiling has (inflated) indices $(M, N)$, then its $x$-coordinate is $\tau(M+\tau N) /(\sqrt{2+\tau})$. It corresponds to a number of letters $M+N$. In the superspace representation the point $\mathbf{r}=(M, N)$ is $\left(M \mathbf{e}_{1}+N \mathbf{e}_{2}\right) \tau$. From this the number $M+N$ can be obtained as $\frac{1}{\tau}\left(\mathbf{e}_{1}+\mathbf{e}_{2}\right) \cdot \mathbf{r}$. It follows that $j=\left(\mathbf{r}_{B}-\mathbf{r}_{A}\right) \cdot(1,1) / \tau$. When the tile diffuses to the right, the starting points $A$ must be an endpoint of a letter $s$ of the non-inflated tiling, hence the endpoint of a letter $L$ of the inflated tiling. They are 
thus counted by the letter $N$ and can be obtained from $\mathbf{r}_{A} \cdot(0,1) / \tau$, provided we have well selected the points $\mathbf{r}_{A}$ to be endpoints of letters $L$. When the tile diffuses to the left, the starting points $A$ must be the beginning point of a letter $s$ of the non-inflated tiling. They are therefore also tied up with a letter $L$ of the inflated tiling, counted by the letter $N$ and obtained by $\left(\mathbf{r}_{A}+(1,0)\right) \cdot(0,1) / \tau$, provided we have well selected the points $\mathbf{r}_{A}$ to be endpoints of letters $L$.

This brings us to the selection of points. In the calculation of the Fourier transform $\mathcal{F}\left(\mathcal{C}_{m, j}\right)$, all lattices points of the non-inflated tiling enter into the calculation. They can be obtained by a cut method from the superspace space presentation of the inflated tiling by decorating the nodes with a composite atomic surface $\mathcal{W}_{0}=\mathcal{W}_{1} \cup \mathcal{W}_{2}$ that has two parts: a long one, $\mathcal{W}_{1}$ centered on the node, and a shorter one, $\mathcal{W}_{2}$, at a distance $\sigma$ towards the left, that generates the points of the non-inflated tiling that are not present in the inflated tiling. $\mathcal{W}_{1}=\{0\} \times\left[-\frac{2 \tau+1}{2 \sqrt{\tau+2}}, \frac{2 \tau+1}{2 \sqrt{\tau+2}}\right]$, $\mathcal{W}_{2}=\left\{-\frac{1}{\sqrt{2+\tau}}\right\} \times\left[-\frac{2 \tau+1}{2 \sqrt{\tau+2}}, \frac{1}{2 \sqrt{\tau+2}}\right]$. We call this composite atomic surface thus $\mathcal{W}_{0}$. When the tile diffuses to the right, the points $\mathbf{r}_{A}$ are generated by an atomic surface $\mathcal{W}_{A}^{+} \subset \mathcal{W}_{1}$, which is that part of $\mathcal{W}_{1}$ that is the projection of $\mathcal{W}_{2}$ onto it: $\mathcal{W}_{A}^{+}=\{0\} \times\left[-\frac{2 \tau+1}{2 \sqrt{\tau+2}}, \frac{1}{2 \sqrt{\tau+2}}\right]$. When the tile diffuses to the left, the starting point $A$ must be the beginning point of a letter $s$ of the non-inflated tiling, hence it must be on an atomic surface $\mathcal{W}_{A}^{-}=\mathcal{W}_{2}$. Finally, the points $\mathbf{r}_{B}$ are generated by an atomic surface $\mathcal{W}_{B}=\mathcal{W}_{1}$. This way, each of the sets of points that we have to consider, viz. $\mathbf{r}$ for $\mathcal{C}_{m, j}, \mathbf{r}_{A}$ for $j$ and $m$, and $\mathbf{r}_{B}$ for $j$, build a different kind of $\mathrm{QC}$, that we can note as $\mathbb{Q C}_{0}, \mathbb{Q C}_{A}^{+}, \mathbb{Q C}_{A}^{-}$ and $\mathbb{Q C}_{B}$ respectivily. Each of these has its Fourier transform. The positions of the Bragg peaks in these Fourier transforms will be the same for the four QCs, but their weights (i.e. their amplitudes) will be different. They can be obtained from a calculation of the Fourier transforms $w_{0}=\mathcal{F}\left(\mathcal{W}_{0}\right), w_{A}^{+}=\mathcal{F}\left(\mathcal{W}_{A}^{+}\right), w_{A}^{-}=\mathcal{F}\left(\mathcal{W}_{A}^{-}\right), w_{B}=\mathcal{F}\left(\mathcal{W}_{B}\right)$ of the corresponding atomic surfaces in a standard fashion. We are now ready for the calculation of the stucture factor of Eq. (17).

The Fourier transform of $\chi[A B]$ is $\left(e^{\imath Q_{\|} x_{B}}-e^{\imath Q_{\|} x_{A}}\right) / i Q_{\|}$. This has to be convoluted with the Fourier transform of the QC, which yields:

$$
\sum_{\mathbf{Q}^{*} \in \mathbb{L}} w_{0}\left(\mathbf{Q}^{*} \cdot \mathbf{e}_{\perp}\right)\left(e^{\imath\left(\mathbf{Q}-\mathbf{Q}^{*}\right)_{\|} \cdot \mathbf{r}_{B}}-e^{\imath\left(\mathbf{Q}-\mathbf{Q}^{*}\right)_{\|} \cdot \mathbf{r}_{A}}\right) / i\left(\mathbf{Q}-\mathbf{Q}^{*}\right) \cdot \mathbf{e}_{\|},
$$

where $\mathbf{Q}^{*}$ stands for the $\mathbf{Q}$-values of the (two-dimensional) Bragg peaks and $\mathbb{L}$ is the set of these Bragg peaks. We have $\sin j q=\left(e^{\imath q\left(\mathbf{r}_{B}-\mathbf{r}_{A}\right) \cdot(1,1) / \tau}-e^{-\imath q\left(\mathbf{r}_{B}-\mathbf{r}_{A}\right) \cdot(1,1) / \tau}\right) / 2 \imath$, while $e^{\imath m \psi_{k}}=e^{\imath\left(\mathbf{r}_{A} \cdot(0,1) / \tau\right) \psi_{k}}$. Let us first perform the sum over the configurations for which $x_{A}<x_{B}$. Then $j \geq 0$. For $\mathbf{p}^{(q,+, k)}$ we obtain:

$$
\sum_{\mathbf{r}_{A}, \mathbf{r}_{B}} \sum_{\mathbf{Q}^{*} \in \mathbb{L}}-\frac{w_{0}\left(\mathbf{Q}^{*} \cdot \mathbf{e}_{\perp}\right)}{2\left(\mathbf{Q}-\mathbf{Q}^{*}\right) \cdot \mathbf{e}_{\|}}\left(e^{\imath\left(\mathbf{Q}-\mathbf{Q}^{*}\right)_{\|} \cdot \mathbf{r}_{B}}-e^{\imath\left(\mathbf{Q}-\mathbf{Q}^{*}\right)_{\|} \cdot \mathbf{r}_{A}}\right)\left(e^{\imath q\left(\mathbf{r}_{B}-\mathbf{r}_{A}\right) \cdot(1,1) / \tau}-e^{-\imath q\left(\mathbf{r}_{B}-\mathbf{r}_{A}\right) \cdot(1,1) / \tau}\right) e^{\imath\left(\mathbf{r}_{A} \cdot(0,1) / \tau\right) \psi_{k}}
$$

which splits into 4 terms. The first term is:

$$
\sum_{\mathbf{r}_{A}, \mathbf{r}_{B}} \sum_{\mathbf{Q}^{*} \in \mathbb{L}}-\frac{w_{0}\left(\mathbf{Q}^{*} \cdot \mathbf{e}_{\perp}\right)}{2\left(\mathbf{Q}-\mathbf{Q}^{*}\right) \cdot \mathbf{e}_{\|}} e^{\imath\left(\left[\left(\mathbf{Q}-\mathbf{Q}^{*}\right)_{\|}+q(1,1) / \tau\right] \cdot \mathbf{r}_{B}+\left[(0,1) \psi_{k}-q(1,1)\right] \cdot \mathbf{r}_{A} / \tau\right)}
$$

For this term it is convenient to perform first the sum over $\mathbf{r}_{B}$. Let us first calculate this sum without the condition $x_{A}<x_{B}$. This unconditional sum over $\mathbf{r}_{B}$ is the two-dimensional Fourier transform for a $\mathbf{Q}$-vector $\left(\mathbf{Q}-\mathbf{Q}^{*}\right)_{\|}+$ $q(1,1) / \tau$ of the part of the two-dimensional superlattice that lies in the strip $\mathcal{W}_{B} \times E_{\|}$that is used to define $\mathbb{Q} \mathbb{C}_{B}$. (Here $E_{\|}$is parallel space). The two-dimensional Fourier transform of this strip-restricted superlattice is different from zero only for $\mathbf{Q}$-vectors $\mathbf{G} \in \mathbb{L} \oplus E_{\perp}$, that belong to the set composed of all straight lines, that are perpendicular to the cut and contain a two-dimensional Bragg peak. (Here $E_{\perp}$ is perpendicular space). The intensity is thus non-zero if $\left(\exists \mathbf{Q}^{* *} \in \mathbb{L}\right)(\exists x \in \mathbb{R})\left(\left(\mathbf{Q}-\mathbf{Q}^{*}\right)_{\|}+q(1,1) / \tau=\mathbf{Q}^{* *}+x \mathbf{e}_{\perp}\right.$. The parallel component of $q(1,1) / \tau$ is $q \tau / \sqrt{2+\tau}$. Hence the intensity will be non-zero when $\left(\mathbf{Q}-\mathbf{Q}^{*}\right)_{\|}+q \tau / \sqrt{2+\tau}=\mathbf{Q}_{\|}^{* *}$, such that $\mathbf{Q}_{\|}=\mathbf{Q}_{\|}^{* *}+\mathbf{Q}_{\|}^{*}-q \tau / \sqrt{2+\tau}$. Let us first keep $\mathbf{Q}^{*}$ fixed. Then for each $\mathbf{Q}^{* *}$-value a corresponding $\mathbf{Q}_{\|}$-value can be found that satisfies the condition. This defines thus a set of satellites at a distance $-q \tau / \sqrt{2+\tau}$ from each QC Bragg peak. All these satellites are valid $\mathbf{Q}_{\|}$-values. The term $1 /\left(\mathbf{Q}-\mathbf{Q}^{*}\right) \cdot \mathbf{e}_{\|}$reduces to $1 /\left(\mathbf{Q}_{\|}^{* *}-q \tau / \sqrt{2+\tau}\right)$. The perpendicular component of $q(1,1) / \tau$ is $-q /\left(\tau^{2} \sqrt{2+\tau}\right)$, such that $\left[\left(\mathbf{Q}-\mathbf{Q}^{*}\right)_{\|}+q(1,1) / \tau\right]$ will be at this $Q_{\perp}$ value on the line through $\mathbf{Q}^{* *}$. The amplitude in the Fourier transform for the satellites is thus given by $w_{B}\left(Q_{\perp}^{* *}+q /\left(\tau^{2} \sqrt{2+\tau}\right)\right) /\left(\mathbf{Q}_{\|}^{* *}-q \tau / \sqrt{2+\tau}\right)$. As $\mathbf{Q}^{*}$ also runs through $\mathbb{L}$, each particular satellite is realized once for every value of $\mathbf{Q}^{* *}$. The dominant amplitude contribution to a satellite will come from its realization with $\mathbf{Q}^{* *}=0$, such that in a first approximation the intensity of a satellite is 
proportional to $1 / q^{2}$. $\mathbf{Q}^{* *}=0$ implies that the satellite position $\mathbf{Q}_{\|}$lies close to the Bragg peak at $\mathbf{Q}_{\|}^{*}$, and the factor $w_{0}\left(-\mathbf{Q}^{*} \cdot \mathbf{e}_{\perp}\right)$ makes sure that the intensities of the dominant realizations scale with the intensities of their associated Bragg peaks at $\mathbf{Q}_{\|}^{*}$.

In reality we must perform the sum with the condition $x_{B}>x_{A}$. The condition can be introduced by multiplying the acceptance strip further with a step function along $E_{\|}$at $x_{A}$. Hence we must replace $\mathbb{L} \oplus E_{\perp}$ by its convolution with the Fourier transform of this step function, which is $\delta\left(\mathbf{G}_{\perp}\right) e^{\imath \mathbf{G}_{\|} \cdot \mathbf{r}_{A}} \frac{1}{2}\left[\delta\left(\mathbf{G}_{\|}\right)-\imath /\left(\pi \mathbf{G}_{\|}\right)\right]$. We must thus convolute our spectrum of satellites with this function. The term $e^{\imath \mathbf{G}_{\|} \cdot \mathbf{r}_{A}}$ leads to $e^{\imath\left[\left(\mathbf{Q}-\mathbf{Q}^{*}\right)_{\|}+q(1,1) / \tau\right] \cdot \mathbf{r}_{A}}$, and this enters into the final sum, which becomes a two-dimensional Fourier transform for a $Q$-vector $\left(\mathbf{Q}-\mathbf{Q}^{*}\right)_{\|}+(0,1) \psi_{k} / \tau$ of the part of the superlattice that lies in the full strip that is used to define $\mathbb{Q C}_{A}^{+}$. This will again lead to satellites. However, as a whole continuum of $\psi_{k}$-values lead to the same value of $\lambda$, this will only multiply the $Q$-dependence of the spectrum obtained so far with a constant.

Description of the further calculations. We have now accomplished one small step of the total calculation. We must also perform the sums of the three other terms in Eq. 201. Then we must also perform the calculations for the cases that the tile diffuses to the left. The contributions must be weighted by $e^{ \pm \imath Q \sigma}-1$. We must repeat all this mutatis mutandis for $\mathbf{p}^{(q,-, k)}$ (for which there will be no parameter $\psi$ ). And finally, we must add terms that correspond to the first, second and fourth terms of Eq. (18). In principle those that correspond to this first term will correspond only to elastic scattering: The sum of the components of an eigenvector that does not correspond to $\lambda=0$ is zero, as this eigenvector is orthogonal to the vector $(1,1,1, \cdots 1, \cdots)$ that corresponds to $\lambda=0$. Those that correspond to the third and fourth terms can be expected to be vanishingly small with respect to the other terms. If we assume that all configurations have equal starting probabilities, we must then square the sums obtained this way, and add up all these squares that go with a same value of $\lambda$.

In principle, it should thus be possible to derive the completely rigorous mathematical expression for the coherent quasielastic scattering signal that corresponds to the model. But the complete calculation would be extremely tedious. We will therefore not perform it, because what we have obtained so far is sufficient for the point that we want to make, viz. that the general response function shares many characteristics of the signal observed by Francoual et al. Let us just for the sake of completeness give the three other terms from Eq. (20) that occur at the same level as the "first" term given by Eq. (21). These terms are:

$$
\begin{gathered}
\sum_{\mathbf{r}_{A}, \mathbf{r}_{B}} \sum_{\mathbf{Q}^{*} \in \mathbb{L}} \frac{w_{0}\left(\mathbf{Q}^{*} \cdot \mathbf{e}_{\perp}\right)}{2\left(\mathbf{Q}-\mathbf{Q}^{*}\right) \cdot \mathbf{e}_{\|}} e^{\imath\left(\left[\left(\mathbf{Q}-\mathbf{Q}^{*}\right)_{\|}-q(1,1) / \tau\right] \cdot \mathbf{r}_{B}+\left[(0,1) \psi_{k}+q(1,1)\right] \cdot \mathbf{r}_{A} / \tau\right)} \\
\sum_{\mathbf{r}_{A}, \mathbf{r}_{B}} \sum_{\mathbf{Q}^{*} \in \mathbb{L}} \frac{w_{0}\left(\mathbf{Q}^{*} \cdot \mathbf{e}_{\perp}\right)}{2\left(\mathbf{Q}-\mathbf{Q}^{*}\right) \cdot \mathbf{e}_{\|}} e^{\imath\left([q(1,1)] \cdot \mathbf{r}_{B} / \tau+\left[\left(\mathbf{Q}-\mathbf{Q}^{*}\right)_{\|}+\left((0,1) \psi_{k}-q(1,1)\right) / \tau\right] \cdot \mathbf{r}_{A}\right)} \\
\sum_{\mathbf{r}_{A}, \mathbf{r}_{B}} \sum_{\mathbf{Q}^{*} \in \mathbb{L}}-\frac{w_{0}\left(\mathbf{Q}^{*} \cdot \mathbf{e}_{\perp}\right)}{2\left(\mathbf{Q}-\mathbf{Q}^{*}\right) \cdot \mathbf{e}_{\|}} e^{\imath\left([-q(1,1)] \cdot \mathbf{r}_{B} / \tau+\left[\left(\mathbf{Q}-\mathbf{Q}^{*}\right)_{\|}+\left((0,1) \psi_{k}+q(1,1)\right) / \tau\right] \cdot \mathbf{r}_{A}\right)}
\end{gathered}
$$

In the third and fourth term we must first sum over $x_{A}$ rather than over $x_{B}$. In these terms the fact that $\psi_{k}$ runs through a dense set will turn the total contributions from such terms into a flat (dynamical) background.

Important Conclusions. When we take into account all eigenvalues $\lambda$, we will obtain an intensity with a $1 / q^{2}$ shape close to each Bragg peak, where $q$ is the distance from the Bragg peak. The intensity will scale with the intensity of the Bragg peak. And in the energy domain, the signal will consist of Lorentzians with a width that is of a $D q^{2}$ type.

We must insist with emphasis on the fact that the signals for different $q$-values are all obtained by a summing over the very same configurations of the $Q C$, viz. the set of configurations we described above. The satellites with wave vector $q$ are not at all caused by some wave with wavelength $2 \pi / q$ in the QC, but by a weighting of QC configurations that are devoid of any wave structure with eigenvectors of the dynamical jump matrix. It is thus not at all some correlation in the atomic positions in the QC that brings about the satellites, but the special character of the eigenvectors that are used as weighting functions. These eigenvectors have a wavelike structure due to the fact that the configuration space is the discrete analogon of a manifold assembled from parts that have a topology that is almost translationally invariant. The reader can check that the eigenvectors of the jump model calculated in [20] also show such a wavelike dependence due to the translational invariance of the topology. It is this wavelike behaviour of the eigenvectors that is responsible for the possibility of having satellites, but it depends on the way such eigenvectors couple to the Fourier transforms of the configurations if a trace of this wavelike character will survive and show up in the final result under the form of satellites. 
It is generally believed that a wavelike Fourier component of random-tiling-like structural disorder is needed to provoke the kind of diffuse scattering observed in QCs. In other words that the Fourier components of the diffuse scattering are structural cut ondulations. The diffusing-tile model shows that also this interpretation may not be unique, especially as the diffuse scattering has now been proved to be the signature of very slow kinetics. The fact that the signal is dynamical opens the way for alternative readings of the diffuse scattering.

Our calculation further confirms a point that we already stressed ever so often, viz. that an interpretation of the satellites in terms of phasons is precluded by the fact that the satellites would have to follow the same temperature dependence as the elementary jumps: All characteristic times of our model have the temperature dependence of $\tau_{0}$. If the signal observed in the speckle experiments is due to atomic displacements, then it can at the very best be due to a secondary relaxation of the lattice in the form of small atomic displacements, e.g. in response to the freezing of the phason dynamics. It is quite possible that both contributions (small atomic displacements with a Debye-Waller factor that shows an anomalous temperature behaviour due to a response to the freezing of the phason jumps and a smaller tile diffusion contribution with a Debye-Waller factor which follows the normal temperature behaviour) are simultaneously present in the data.

We may finally note that it is not obvious that in our model the quasielastic intensity pattern would comply with a phason elasticity Ansatz. (But it is possible to think of variants of our model, e.g. by introducing different jump times between $\mathcal{C}_{0}$ and $\mathcal{C}_{m, 1}$, and this way modulating the probabilities of the branches of the star with the $x_{\perp}$ coordinate of $\mathbf{r}_{A}$. The eigenvalue problem would still remain exactly solvable).

For all these reasons, it is not necessary to pursue our calculations of this diffusing-tile model any further. In fact, in the third paragraph of subsection III.F about conflicting time scales we pointed out that the kind of model we have developed here is physically unrealistic: If one wanted to impose such a model, one would need to introduce a notion that the QC is highly stable with respect to tile flips, in order to validate the assumption that there would be only one flipped tile at a time within the whole QC. A real system will contain many tile flips simultaneously. The prolificity of these flips, with their high hopping rates, raises the question if the long-time persistence of correlations between configurations as observed in our model can survive in a more realistic setting.

The aim of our model is therefore not to give an alternative interpretation of the data but rather to give a counter example to show that the interpretation of the data according to reference [1] is not unique and that it contains unjustified tacit assumptions: (1) that the presence of satellites would be a proof for the presence of a wavelike modulation within the structure, (2) that phason elasticity would be in 1-1-correspondence with phason dynamics, and (3) that one could ignore the fact that the temperature dependence observed in the quasielastic neutron scattering results precludes any interpretation of the diffuse scattering data in terms of tile flip dynamics. Note that the unjustified introduction of correlations in the atomic positions on the basis of (1) is only a first ingredient for the introduction of the wrong paradigm of dynamical phason waves, a second one being the erroneous identification of these correlations in the atomic positions with unphysical long-distance correlations between atomic jumps.

\section{The presence of satellites at wave vector $q$ is not a proof for a presence of a modulation wave with wavelegth $2 \pi / q$.}

This conclusion from model 3 about the invalidity of tacit assumption (1) can be generalized to all jump models. The algorithm to calculate a coherent quasielastic signal for a model of atomic jump dynamics is based on a set of coupled differential equations expressed in terms of a jump matrix, that transcribes the original problem into a model of jumps between configurations. To solve the differential equations one must find the eigenvalues and eigenvectors of the jump matrix. These eigenvectors have a component for each configuration. The components of an eigenvector that corresponds to an eigenvalue $\lambda$ must be used to weight the Fourier transforms of the corresponding configurations. These weighted Fourier transforms are added up such as to yield the amplitude of the structure factor that goes with the eigenvalue $\lambda$. As the very same set of configurations comes into play for different eigenvalues, it is meaningless to want to associate a wavelength or a particular wave pattern in the QC structure with the structure factor of some eigenvalue: The coupling of a wavelength or wave pattern with an eigenvalue would mean that each eigenvalue is associated with its own typical configuration, while the truth is that each eigenvalue is coupled to the same (full) set of configurations, with a different weighting procedure, defined by the components of the corresponding eigenvector. Hence any relationship between a measured "wave vector" $q$ and a special relaxation time $\tau_{q}$, such as e.g. $1 / \tau_{q} \propto q^{2}$, can only originate in the weighting procedure, not in a modulation wave pattern with wave vector $q$, that would exist within the structure. The calculation of model 3 may serve as a worked-out illustration of this argument, rendering it less abstract, but the argument is truly generally valid for all jump models. The weighting factors correspond to a probability formalism for the configurations. We can thus summarize the situation by stating that the signal for a value of $q$ does not correspond to a configuration of the QC but to an invariant, equilibrium probability distribution on the set of all possible configurations of the QC. (As such an equilibrium probability distribution never resumes to a single configuration with probability 1, the system can act up to the probability distribution only by fluctuating indefinitely). Therefore the "wave vector" $q$ does not correspond to a single configuration with a wavelike 
pattern on the physical structure of the QC. At the very best it can correspond to a probability wave on the set of configurations, like it is the case in model 3, where the presence of these waves is due to some translational invariance on the configuration manifold. In more complicated models even this abstract kind of translational invariance can be absent. The dynamical signal is thus even not due to a density wave as discussed in Section II for static diffuse scattering: It is only a probabilistic Van Hove correlation between configurations at time 0 and configurations at a later time $t$. We will now illustrate the point that the presence of satellites does not prove a presence of correlations in the even more salient example of single-vacancy diffusion.

\section{Single-vacancy diffusion}

Let us consider the problem of the diffusion of a single vacancy on the Fibonacci chain. The probabilities $p_{j}$ that the vacancy is located at site $j$, are governed by the jump equations $(\forall j \in \mathbb{Z})\left(\frac{d p_{j}}{d t}=-\frac{1}{\tau_{j-1, j}}\left(p_{j}-p_{j-1}\right)+\frac{1}{\tau_{j, j+1}}\left(p_{j+1}-p_{j}\right)\right)$, where the relaxation times $\tau_{j, j+1}=\tau_{L}$ if the bond between atoms $j$ and $j+1$ is long, and $\tau_{j, j+1}=\tau_{S}$ if it is short. We can compare this with the dynamical equations for the atomic displacements $u_{j}$ in the lattice dynamics, when only first-neighbour intercations are considered: $(\forall j \in \mathbb{Z})\left(\frac{d^{2} u_{j}}{d t^{2}}=-k_{j-1, j}\left(u_{j}-u_{j-1}\right)+k_{j, j+1}\left(u_{j+1}-u_{j}\right)\right)$, where the spring constants $k_{j, j+1}=k_{L}$ if the bond between atoms $j$ and $j+1$ is long, and $k_{j, j+1}=k_{S}$ if it is short. We see that apart from the specific values of the constants $\tau_{L}, \tau_{S}$, and $k_{L}, k_{S}$, the sets of equations have the same structure, such that the eigenvalue problems of the jump matrix and the dynamical matrix are identical.

In the case of the jump matrix the eigenvalue $\lambda$ and the corresponding eigenvector $\mathbf{v}^{(\lambda)}$ will be used to find a solution $\mathbf{p}=e^{-t / \tau_{\lambda}} \mathbf{v}^{(\lambda)}$, such that $-1 / \tau_{\lambda}=\lambda$. In the case of the dynamical matrix the eigenvalue $\lambda$ and the corresponding eigenvector $\mathbf{v}^{(\lambda)}$ will be used to find a solution $\mathbf{u}=e^{i \omega_{\lambda} t} \mathbf{v}^{(\lambda)}$, such that $-\omega_{\lambda}^{2}=\lambda$. The solution of such eigenvalue problems on the Fibonacci chain is horrendously difficult. Apart for $\lambda=0$, the solutions are in general not quasiperiodic. We should thus not expect eigenvector solutions in the form of Bloch waves $v_{j}^{(\lambda)}=e^{\imath q j}$. At the best, we can attempt a Fourier decomposition of the eigenvectors into waves, $v_{j}^{(\lambda)}=\sum_{q} a_{q}^{(\lambda)} e^{\imath q j}$. Nevertheless, in the long-wavelength limit for the phonon problem the distribution of $q$-values will be extremely narrow and the dominant Fourier component of $v^{(\lambda)}$ will be $w_{j}^{(\lambda)}=e^{\imath q j}$, with a linear relationship $\omega=c q$, that defines the speed of sound $c$. This is at least what is observed experimentally in all real QCs. From the analogy it will then follow that in the long-wavelength limit for the vacancy diffusion problem the dominant Fourier component of the eigenvector will also follow a linear relationship $1 / \tau=D q^{2}$, that defines the vacancy diffusion constant. When $k_{L}=k_{S}$ and $\tau_{L}=\tau_{S}$ the eigenvectors are true waves and these results become exact.

In the problem of the lattice dynamics we will calculate the amplitude of the coherent neutron scattering signal for a neutron momentum transfer $Q$ as $\sum_{j \in \mathbb{Z}} e^{\imath Q\left(x_{j}+u_{j}\right)}$, where $x_{j}$ is the equilibrium position of atom $j$. To first order of a Taylor series expansion this can be developed as $\sum_{j \in \mathbb{Z}} e^{\imath Q x_{j}}\left(1+\imath Q u_{j}\right)=\mathbb{L}_{Q}+\imath Q \sum_{j \in \mathbb{Z}} v_{j}^{(\lambda)} e^{\imath Q x_{j}} e^{\imath \omega_{\lambda} t}$. Here the first term $\mathbb{L}_{Q}$ is the elastic signal in the form of the spectrum of Bragg peaks as a function of $Q$. The second term is the inelastic contribution. Of course for $u_{j}$ we have taken $v_{j}^{(\lambda)} e^{\imath \omega_{\lambda} t}$.

In the problem of single-vacancy diffusion the configuration space can be put into 1-1-correspondence with the sites of the lattice. The configuration $\mathcal{C}_{j}$ will be the one where the vacancy is situated at the lattice site $j$. Its Fourier transform $\mathcal{F}_{Q}\left(\mathcal{C}_{j}\right)$ will be given by $\mathbb{L}_{Q}-e^{\imath Q x_{j}}$. The amplitude of the coherent neutron scattering signal for a neutron momentum transfer $Q$ is then given by $\sum_{j \in \mathbb{Z}} p_{j} \mathcal{F}_{Q}\left(\mathcal{C}_{j}\right)=\sum_{j \in \mathbb{Z}} v_{j}^{(\lambda)}\left(\mathbb{L}_{Q}-e^{\imath Q x_{j}}\right) e^{-t / \tau_{\lambda}}$. The first term is of the form $\mathbf{v}^{(\lambda)} \cdot \mathbf{v}^{(0)} \mathbb{L}=\delta(\lambda) \mathbb{L}$, as for $\lambda=0$ the eigenvector is of the form $[1,1,1 \cdots 1,1, \cdots]$. When $\lambda=0$ we recover the elastic signal as $\delta \cdot \mathbb{L}-\mathbb{L}$, where weight of the second part is negligible with respect to that of the Dirac measure $\delta$. For $\lambda \neq 0$ we recover the inelastic signal. We can thus compare the $Q$ dependences for the dynamical parts of the amplitudes of the signals:

$$
\imath Q\left(\sum_{j \in \mathbb{Z}} v_{j}^{(\lambda)} e^{\imath Q x_{j}}\right) e^{\imath \omega_{\lambda} t}[\text { phonons }] \Leftrightarrow-\left(\sum_{j \in \mathbb{Z}} v_{j}^{(\lambda)} e^{\imath Q x_{j}}\right) e^{-t / \tau_{\lambda}} \quad[\text { vacancy }] .
$$

Hence, apart from a factor $Q^{2}$, the $Q$-dependences of the inelastic signals for the phonon dynamics and for the vacancy diffusion have for a given eigenvalue $\lambda$ the same structure. In the long-wavelength limit this $\mathrm{Q}$-dependence is coupled to phonon waves $e^{\imath c \hbar q t}$ and to relaxation times $e^{-D q^{2} t}$, where $D$ is the vacancy diffusion constant. In the energy domain $e^{\imath c \hbar q t}$ will lead to an inelastic delta peak at non-zero energy $c \hbar q$ and of zero width, while $e^{-D q^{2} t}$ will lead to an inelastic Lorentzian peak centered at zero energy and of width $\hbar D q^{2}$. (Because the inelastic signal is centered at zero energy it is quasielastic). In the long-wavelength approximation $v_{j}^{(\lambda)} \approx w_{j}^{(\lambda)}=e^{\imath q j}$, and through $q j=q(1,1) \cdot \mathbf{r}_{j}$ in superspace, the $Q$-dependence leads to satellites at distance $q / \sqrt{2+\tau}$ from each Bragg peak. It is interesting to note that when $k_{L}=k_{S}$ and $\tau_{L}=\tau_{S}$ these results become exact, even for large wave vectors. 
Single-vacancy mediated atomic jump diffusion is a school example by exellence of what in folk lore is being considered as an "incoherent process", and the calculation of the incoherent signal for this process is presented in many text books. Our calculation illustrates very clearly the falsehood of the wide-spread belief that the incoherence or coherence of a scattering process or signal would stand in 1-1-correspondence with the incoherence or coherence of the physical process in the sample that is being probed by it: The calculation yields a coherent signal in the form of a set of satellites at a distance $q$ from the Bragg peaks, while there is no correlation between the atomic jumps whatsoever. When we limit ourselves to first-neighbour models, the previous argument can be easily generalized to two- and three-dimensional QCs.

\section{Fluctuating Fourier components}

Imagine that we have a lattice where a single atom has been displaced over a distance $\mathbf{u}$ from its initial place $\mathbf{r}_{j}$. Call the Fourier transform of the perfect lattice $\mathcal{F}(\mathbf{Q})$. The Fourier transform of the lattice with the single defect will then be $\mathcal{F}(\mathbf{Q})+e^{\imath \mathbf{Q} \cdot\left(\mathbf{r}_{j}+\mathbf{u}\right)}-e^{\imath \mathbf{Q} \cdot \mathbf{r}_{j}}$. This can be expanded in a Taylor series as $\mathcal{F}(\mathbf{Q})+e^{\imath \mathbf{Q} \cdot \mathbf{r}_{j}}\left[\imath(\mathbf{Q} \cdot \mathbf{u})-\frac{1}{2}(\mathbf{Q} \cdot \mathbf{u})^{2} \ldots\right]$. This signal would be detected by coherent scattering. Hence it follows that for all wave vectors $\mathbf{Q}$ (except those in the plane defined by $\mathbf{Q} \cdot \mathbf{u}=0$ ) the intensity changes. At every reciprocal-lattice point $\mathbf{Q}$ (that does not belong to the plane) there is a change of intensity. This idea can be generalized to a kind of pertubation theory that permits to get some idea about the changes of the static signal, when a change of the position of many atoms is at stake. Does the observation of such changes (on a characteristic time scale $\tau_{0}$ ) mean that there are dynamical modes with wave vector $\mathbf{Q}$ on the characteristic time scale $\tau_{0}$ within the system? Certainly not. We must emphasize that what we just described is not what is being measured by photon correlation spectroscopy. What is being measured involves correlations between two such intensities at two different times: The measurement of an intermediate scattering function is more subtle than just a measurement of the fluctuating static structure facor. Photon correlation spectroscopy is a correct measurement of the dynamics and the intermediate scattering function. But the paradigm we just described is exactly what has been used to analyze the data: The fact that the diffuse scattering can be Fourier analyzed through a superspace description (in terms of physically meaningless density waves) and that it exihibits slow kinetics (on a time scale that forbids any confrontation with dynamical data) has been used to claim that dynamical phason modes have been observed in QCs. From the calculation one can see that the major changes in intensity will be roughly speaking proportional to $\mathcal{F}(\mathbf{Q})$, due to the cross products between $\mathcal{F}(\mathbf{Q})\left(\right.$ and $\left.\mathcal{F}(\mathbf{Q})^{*}\right)$ and the other, smaller terms. The terms that do not contain $\mathcal{F}(\mathbf{Q})$ (and $\mathcal{F}(\mathbf{Q})^{*}$ ) can be neglected in this approach. Hence the intensities which will fluctuate the most under any change of the system that is small enough to warrant such a pertubational approach will be the diffuse scattering and the Bragg peaks. They will thus no doubt lead to the most prominent features in the measured correlation function. Even in the wrong paradigm of the static structure factor approach the fluctuating speckle would not prove that it is the phason modulation that is (hypothetically) observed in the static signal that fluctuates. In reality, the signal must be analyzed in a completely different fashion as we have shown on the basis of our model calculations in the preceding subsubsections. The fact that the speckle signal fluctuates is therefore not at all a proof that a phason modulation field, claimed to cause the diffuse scattering, would exist and fluctuate, as has been claimed. The few additional arguments that have been advanced to strengthen the credibility of this claim have been analyzed above. They are not convincing. One cannot study dynamics on the basis of a few selected points $\mathbf{Q}$, in reciprocal space and interpret the intensities in these points as though they were produced by the same physical causes as the intensity of the elastic structure factor at the same values $\mathbf{Q}$. To identify the dynamical process one must measure the dynamical structure factor over the whole of reciprocal space and confront it with an appropriate calculation. Simultaneously to this unjustified claim that "dynamical phason modes" would have been observed, the doctrine has been published that the definition of phason dynamics must be changed in such a way that our work on phason hopping can be dismissed as a true observation of phason dynamics (see below).

\section{LACK OF PROOF FOR THE RANDOM TILING MODEL}

\section{A. Introduction}

In reference 24 the authors stated that the temperature dependence of the data are "in contradiction with the hypothesis of a simple random tiling model". They added that the tile flip interpretation can be maintained by introducing a more complex random tiling model. 17] The random tiling model and the tile flip scenario can thus be claimed on the basis completely opposite temperature behaviours. This is thus not very convincing evidence for the random tiling model. To cite Sir Karl Popper: "Falsifiability should be the criterion of demarcation in science". 25] Moreover, as already pointed out, all the temperature data might show is that there is some softening of the elastic constants. What the temperature dependence settles within the context of the random tiling model, is that the pristine model should be abandoned in favour of Widom's model. 17]

The diffuse scattering data, without a mention of their temperature dependence, were eventually reported in 
reference [26]. Nonetheless, in the final discussion of that paper, the authors already introduce the arguments to thwart the criticism that can be formulated based on the temperature behaviour observed. As the reader had no access to the information about the temperature dependence, he could not possibly understand the issues at stake. Rather than the mention of a crucial problem raising serious doubts 27] about the validity of the interpretation, it looked like a very puzzling digression.

In that paper, the authors concluded that their data were "compatible" with the random tiling model. The problem with this statement does not reside in what it literally states, but in its tacit tertium non datur. It does not say that the data may also be compatible with other models. Due to this formulation the reader may pick up the totally unjustified belief that the data would have conclusively proved the random tiling model. As a matter of fact, such overinterpretations have indeed made their way to the literature. 28]. This is of course very harmful. It has installed a strong oral tradition, that made it very difficult to point out the absence of convincing proof for the random tiling model. The unassailable character of the literal interpretation of the formulation may have contributed to this situation. It is not by qualifying this problem as an old story, that the validity of these claims can be settled.

\section{B. The data claimed to be evidence are too unspecific}

At this point it is perhaps good to review the evidence the authors proposed in favor of their interpretation in terms of tile flips.

(a) An $1 / q_{\|}^{2}$ dependence of the intensity. This is unspecific and occurs in many other cases of Huang scattering in crystals. 29]

(b) A certain shape of the diffuse scattering intensity contours. Again, this is unspecific, and purely due to symmetry in analogy with the situation in conventional crystals. 29] Such symmetry-based arguments do not contain any information about the underlying mechanisms or interactions.

(c) The shapes of the contours depend only on the phason-phason elastic constants. This is the only feature that is not unspecific. As such, it may rule out a number of alternative models. But this does not mean that it rules out all possible alternative models. We have clearly shown that it is still not unique, even if it has been presented as self-evident that it would be unique.

As the temperature dependence of the neutron scattering data does not agree with an interpretation in terms of tile flips, we actually know that the correct model must be different.

We should insist on the fact that an interpretation in terms of tile flips is a derived application of the elasticity theory, which is formulated in terms of a continuum of small atomic displacements rather than on a discrete set devoid of infinitesimals. The very definition of an elastic constant cannot be written down if we cannot assume that the atomic displacements explore a continuum. The validity of the derived application is not obvious, as on the microscopic level tile flips do not explore a continuum of atomic displacements. This is very different from the continuum limit for phonons. There is thus a priori no good theoretical rationale to explain the results of Tang et al. 10, 11] except the post facto observation that it works despite such theoretical objections.

To improve on this situation, Henley [30] has proposed an argument of coarse graining. Unfortunately, this may rather have contributed to the fact that it was not realized that the interpretation of the data is not unique. Without this argument of coarse graining, the results of Tang et al. 10, 11] could have stood out as a clearly different alternative in the form of a discrete displacement field rather than a continuous one.

In any case, it remains a cracking pass to generalize the finding by Tang et al. 10, 11] in the sense that one takes it for granted that the only exclusive way to obtain such a dependence on the phason elastic constants would be the tile flips that are so improper for the first-hand application of the theory. Similarly, in Widom's [1] Landau-type theory it is not granted that the phason elastic constants can only correspond to tile flips: Even if some elastic instability were observed in diffraction experiments that completely tallied with his calculations, it would not yet prove the random tiling scenario.

We may note that the phason elasticity constants obtained by the authors of their diffuse scattering data do not agree with calculations based on canonical cell tilings. 31]

Finally, we must point out that the alternative that the diffuse scattering could be due to chemical disorder has not been properly ruled out. If the data really were due to chemical disorder, than they would not contain any proof for the random tiling model.

In conclusion, we think that the experimental evidence is too narrow to claim that it would be a proof for the validity of the random tiling model. It could be misleading to want to harness the observation of diffuse scattering into a stability issue in the form of an opposition between random tiling models and a perfect quasicristal model. 


\section{Absence of proof for a phase transition}

We may note that the QC-crystal transition postulated by the random tiling model is of first order, while in the example of $\mathrm{NaNO}_{2}$ cited in reference [1], as a paradigm for the observations, the transition is second order.

We may also note that there is no proof for the existence of the phase transition postulated by the random tiling model. In fact, one must introduce ad hoc assumptions in order to deny the clear experimental evidence that there is no phase transition. One might well invoke the superstructure reported by Ishimasa, 32] which shows both satellite peaks and diffuse scattering. However, for this exceptional observation there are many other ones where there is no phase transition at all. 33. But even in the favorable case reported by Ishimasa, the dominant diffuse scattering maxima are centered on the Bragg positions of the QC, not on the satellites. The scenario is thus not one of diffuse scattering progressively building up at the future satellite positions, and eventually turning into satellite Bragg peaks.

Let us finally point out that in a discussion about the presence of a phase transition or otherwise, only those who claim such a transition exists can solve the issue by providing the proof. It is in fact virtually impossible to prove that there is no phase transition: absence of proof can simply not be taken as proof of absence.

\section{VIII. "PHASON JUMPS" IS BY ALL CRITERIA AN APPROPRIATE TERMINOLOGY}

It has been claimed that the terminology "phason jumps" would not be appropriate, and that the terminology "phason" should be reserved to the modes postulated in Eq. [42] in the paper by Janssen et al. 4] There are several problems with this restriction or change of definitions:

(1) Before defining "phason modes" as is done in reference[4], one could wish to wait until convincing proof of their existence has been given. The "phason modes" are just a conjecture. We have seen that they are introduced on the basis of an argument that is not rigorous. It has to be checked that the definition is meaningful. It rather seems as though this is not the case.

(2) To motivate the claim mentioned, it has been argued that phason jumps are not characteristic of quasicrystals because they also occur in 1/1 approximants. 34 This capitalizes on undue remarks in references [35] where it was stated that phason jumps are "not a special property for QCs". These remarks focused on the fact that extremely fast jumps also occur in a B2-based phase that can contain up to $12 \%$ vacancies. But phason jumps are not only "special" in just being fast, and it is doubtful that the presence of atomic jumps in a very unusual sample with an exceptional amount of vacancies could shed light on such issues. What is obvious is, that if atomic jumps occur in a system, they will forcedly produce diffuse scattering, and that diffuse scattering can occur for many other reasons. Diffuse scattering or speckle are certainly even less characteristic of quasicrystals. And the modes that are supposed to replace the phason jumps as the unique observation of phason dynamics most probably just do not exist.

(3) The terminology "phason" was introduced by Overhauser 36] in the field of dynamics of charge-density waves. It did not rely on elasticity considerations. It is thus the terminology "phason elasticity" that is a derived concept, rather than the phason jumps.

(4) The argument that "phason jumps" is not a good terminology has been pushed to the extreme. One has even proposed a sarcastic, belittling terminology "flipon" for them, [6, 37] but e.g. Edegawa et al. have clearly pointed out that the terminology is perfectly appropriate. [6]

\section{CONCLUSION}

In conclusion, we can resume the situation as follows. The diffraction pattern of a QC allows for a Fourier decomposition in superspace based on (physically meaningless) static density waves. The Fourier components that describe the diffuse scattering in this Fourier decomposition can be interpreted as static modulation waves in superspace. When there are fluctuations in the QC, automatically the intensities of all these Fourier components will change, i.e. the intensities of all Fourier components will fluctuate. Such fluctuations of the intensity of a Fourier component do of course not imply that the Fourier component would be a dynamical wave, even not in superspace. It is just that the contribution of the Fourier component to the diffraction pattern fluctuates, whenever the diffraction pattern fluctuates, whatever the kind of fluctuation might be. What the authors measure is not such a fluctuation of the Fourier components, but a component of the intermediate scattering function. Nevertheless, their interpretation of the Q-dependence of the intermediate scattering function follows exactly the mental image of such a fluctuation of a Fourier component of the static structure factor, as it interprets the intermediate scattering function using the modulation waves from the analysis of the static structure factor. Although a dynamical structure factor cannot be analyzed as a static structure factor, the authors start from an Ansatz of dynamical waves on the basis of the superspace modulation picture which is only valid for the static structure factor analysis. As the topology and the translational invariance of the network of the bonds in the superspace crystal (which are part of the mechanism needed to justify such an assumption of dynamical waves) are destroyed by the introduction of the cut, it is physically wrong 
to carry over the Ansatz of dynamical waves from the virtual crystal in superspace to the real QC in physical space. This error has profound physical implications. Even if one were to allow for this error, there is a whole series of secondary objections against such an interpretation: (1) Even within the context of a wave Ansatz it is not unique, e.g. chemical disorder or a field of small atomic displacements can lead to the same result. These alternatives are more physical and they have not been properly excluded. (2) A closer look at them reveals that the hypothetical waves are all but waves, and their hypothetical wavelengths all but wavelengths as can clearly be seen in the limit of small amplitudes. (3) The idea of a phason wave is conceptually wrong because it amalgamates without any discussion two conflicting physical pictures (propagating phonons and diffusing atomic jumps) and introduces unphysical long-distance correlations between atomic jumps by confusing correlations between atomic positions with correlations between atomic jumps. It uses an interpretation of a static structure factor to analyze a dynamical structure factor. (4) In order to justify certain approximations, the model of the authors requires that one assumes that the phason wave has a small amplitude while it is not mentioned that in the limit of very small amplitudes long-range diffusion is locked. (5) The model associates a phonon-like wavelength with a $q$-vector while the discrete character of these $q$-values is not observed in the form of clear isolated inelastic peaks as for phonons, and $q$ can have a completely different meaning than a wave-length. (6) The presence of such discrete satellites does not require any presence of a physical wave, which further emphasizes how the interpretation that has been proposed is not at all unique. (7) The Ansatz is contradicted by the temperature behaviour observed in neutron scattering studies whose interpretation is much more reliable and open to cross-checking by other methods, in sharp contrast with the data of the authors which are unassailable. (8) In passing, the authors have confused (a) coherent scattering signals as evidence for the presence of correlations and (b) mathematical Fourier components as evidence of physical waves.

Hence, it is not true that the experimental data would be evidence for the existence of phason waves in QCs, while from a conceptual and theoretical viewpoint the introduction of such a notion of phason waves is awkward.

[1] S. Francoual, F. Livet, M. de Boissieu, F. Yakhou, F. Bley, A. Létoublon, R. Caudron, and J. Gastaldi, Phys. Rev. Lett. 91, 225501 (2003); A. Létoublon, F. Yakhou, F. Livet, F. Bley, M. de Boissieu, L. Mancini, R. Caudron, C. Vettier and J. Gastaldi, Europhys. Lett. 54, 753 (2001).

[2] M.V. Jarić and D.R. Nelsson, Phys. Rev. B 37, 4458 (1988).

[3] T.C. Lubensky in Introduction to Quasicrystals, ed. by M.V. Jarić, (Boston Academic Press), 1988.

[4] T. Janssen, O. Radulescu, and A.N. Rubtsov, Europhys. J. B 29, 85 (2002).

[5] K. Edagawa, K. Suzuki, and S. Takeuchi, Phys. Rev. Lett. 85, 1674 (2000).

[6] C.L. Henley, M. deBoissieu, and W. Steurer, Phil. Mag. 86, 1131 (2006); see paragraph 4.1.1. on p.1145 (chemical disorder), bottom of p.1142 (Fourier component), Question 2 on p.1143 ("flippon").

[7] D. M. Frenkel, C. L. Henley, and E. D. Siggia, Phys. Rev. B 34, 3649 (1986); L. S. Levitov, Quasicrystals, The State of the Art, ed. D. P. DiVincenzo and P. J. Steinhardt, Directions in Condensed Matter Physics, Vol. 11, (World Scientific, Singapore, 1991) pp.239; A. Katz, Proceedings of the Anniversary Adriatico Conference on Quasicrystals, ICTP, Trieste, Italy, 4-7 July, 1989, ed. M.V. Jarić and S. Lundqvist, (World Scientific, Singapore, 1989), p.200; A. Katz, Proceedings of the Workshop "Number Theory in Physics", Les Houches, France, 1989, ed. J.M. Luck, P. Moussa, M. Waldschmidt, and C. Itzykson, Springer Proceedings in Physics 47 (Springer, Berlin, 1990), p.100. See however also reference [8].

[8] For examples of QC with continuous phasons, see e.g. P. Kalugin and L. S. Levitov, Int. J. Mod. Phys. B 3, 877 (1989). M. Wirth and H.-R. Trebin, Europhys. Lett. 13, 61 (1990). These counter examples jeopardize the possibility to define QC unambiguously on the basis of the non-continuous nature of the phasons.

[9] P.M. Dewolff, T. Janssen, and A. Janner, Acta. Crtyst. A 37, 625 (1981).

[10] L.H. Tang, Phys. Rev. Lett. 64, 2390 (1990).

[11] L.J. Shaw, V. Elser and C.L. Henley, Phys. Rev. B 43, 3423 (1991).

[12] M. de Boissieu et al., at Workshop on Aperiodic Structures, Krakow, Poland, 1-5 July 1996.

[13] R. Bellissent, private communication (1998).

[14] We calculate the Fourier transform within the cut formalism, which is more general than the projection formalism.

[15] M. Duneau, private communication, (2004).

[16] W. Steurer, Z. Kristallogr. 215, 323 (2000). However, it is in general not rigorous to introduce the notion that there exists a 1-1-mapping between a quasicrystal and a periodic structure.

[17] M. Widom, Phil. Mag. Lett. 64, 297 (1991).

[18] G. Coddens, R. Bellissent, Y. Calvayrac and J.P Ambroise, Europhys. Lett. 16, 271(1991).

[19] G.G. Naumis, Chumin Wang, M.F. Thorpe and R.A. Barrio, Phys. Rev. B 59, 14302 (1999).

[20] G. Coddens, J. Phys. I France 4, 921(1994).

[21] P.A. Kalugin and A. Katz, Europhys. Lett. 21, 921 (1993).

[22] G. Coddens, Phys. Rev. B 63, 064105, (2001).

[23] G. Coddens, Eur. Phys. J. B 31, 533 (2003).

[24] M. de Boissieu, M. Boudard, and C. Janot, in ISIS 1994-1995, (Rutherford Appleton Laboratory), p. A23. The authors 
report here their data obtained on the instrument SXD (July 1992).

[25] K.R. Popper in The Logic of Scientific Discovery, 9th edition, (Hutchinson, London, 1977), p.40.

[26] M. de Boissieu, M. Boudard, B. Hennion, R. Bellissent, S. Kycia, A. Goldman, C. Janot, and M. Audier; Phys. Rev. Lett. 75, 89 (1995).

[27] G. Coddens, Int. J. Mod. Phys. B 7, 1679 (1997).

[28] V. Elser, Phil. Mag. B 73, 641 (1996); C.L. Henley, oral presentation at the NATO Advanced Study Institute on Mathematics of Long Range Aperiodic Order. The Fields Institute of Research in Mathematical Sciences, Waterloo, Ontario, Canada, August 21 - September 1, 1995.

[29] A.M. Bratkovsky, S.C. Marais, V. Heine and E.K.H. Salje, J. Phys.: Cond. Matter 6, 3679 (1994).

[30] C.L. Henley in Quasicrystals, The State of the Art, (World Scientific, Singapore, 1991), p. 429.

[31] C.L. Henley, private communication (2005) and Phil. Mag. 86, 1123 (2006), on page 1127.

[32] T. Ishimasa, Y. Fukano, and M. Tsuchimori, Phil. Mag. Lett. 58, 157 (1988).

[33] M. Boudard, M. de Boissieu, A. Létoublon, B. Hennion, R. Bellissent and C. Janot, Europhys. Lett. 33, 199 (1996).

[34] M. de Boissieu, Phil. Mag. 86, 1115 (2006); see page 1117.

[35] J. Dolinšek, T. Apih, P. Jeglič, M. Feuerbacher, M. Calvo-Dahlborg, U. Dahlborg and J.M. Dubois, Phys. Rev. B 65, 212203 (2002); U. Dahlborg, W.S. Howells, M. Calvo-Dahlborg, and J.M. Dubois, J. Phys.: Cond. Matter 12, 4021 (2000).

[36] A.W. Overhauser, Phys. Rev. B 3, 3173 (1971).

[37] M. de Boissieu, M. Boudard, S. Kycia, A.I. Goldman, B. Hennion, R. Bellissent, M. Quilichini, R. Currat and C. Janot in Proceedings of the 5th International Conference on Quasicrystals, Avignon, France, May 22-26, 1995, (World Scientific Singapore, 1996 , p. 577 ; see p. 581. 